\title{
Two-step Procedure Based on the Least Squares and Instrumental Variable Methods for Simultaneous Estimation of von Bertalanffy Growth Parameters
}

\author{
Ivelina Yordanova Zlateva, Technical University of Varna, Varna, Bulgaria
}

Nikola Nikolov, Technical University of Varna, Varna, Bulgaria

\begin{abstract}
Advanced in the present article is a Two-step procedure designed on the methods of the least squares (LS) and instrumental variable (IV) techniques for simultaneous estimation of the three unknown parameters $\mathrm{L} \infty, \mathrm{K}$ and $\mathrm{t} 0$, which represent the individual growth of fish in the von Bertalanffy growth equation. For the purposes of the present analysis, specific MATLAB-based software has been developed through simulated data sets to test the operational workability of the proposed procedure and pinpoint areas of improvement. The resulting parameter estimates have been analyzed on the basis of consecutive comparison (the initial conditions being the same) between the results delivered by the two-step procedure for simultaneous estimation of $\mathrm{L} \infty, \mathrm{K}$ and $\mathrm{t} 0$ and the results obtained via the most commonly employed methods for estimating growth parameters; first, use has been made of the Gulland-and-Holt method for estimating the asymptotic length Lo and the curvature parameter $\mathrm{K}$, followed by the von Bertalanffy method for estimation of $\mathrm{t} 0$.
\end{abstract}

\section{KEYWORDS}

Asymptotic Length, Biological Objects, Fish, Gulland-Holt Plot, Individual Growth, MATLAB, Modeling, Parameter estimates, System Identification

\section{INTRODUCTION}

Determining the fish growth parameters plays a fundamental role not only in the correct detection of the fish stock age and size structure, but also in the process of stock assessment procedure, studies of the population dynamics and sustainable stock management in terms of fish stock exploitation restrictions or specific recommendations for minimum limits on the size (length) of species to be caught legally, selectivity of the fishing gears and equipment, etc. Historic data on growth parameters estimates has been successfully used as a basis for analysis of the stock biological development over time in addition to the analysis of the impact of environmental factors - food availability, variability in specific environmental conditions, such as water temperature, the concentration of dissolved oxygen, the types of species interactions and others. Several functions have been developed to model the growth of fish (Gompertz growth model, Schnute-Richards, logistic, etc.), however the von Bertalanffy model is the most popular and is in the scope of the present material.

The von Bertalanffy mathematical model of individual-based approach to predicting fish growth expresses the length $(L)$ as a function of the age of the fish $(t)$ : starting on February 4, 2021 in the gold Open Access journal, International Journal of Agricultural and Environmental Information Systems 


$$
L(t)=L_{\infty}\left[1-\exp \left(-K\left(t-t_{0}\right)\right)\right]
$$

where: $t$ is the age of the respective fish, $L_{\infty}$ - is the asymptotic length (or mean length of the cohort at age equal to infinity when the study refers to population dynamics), $K$ is a curvature parameter which shows how fast a given individual approaches its asymptotic (finite) length, $t_{0}-$ is the initial condition parameter (determining the point in which the length of the fish is $=0$ ) (Cadima, 2003, Sparre \& Venema, 1998).

Equation (1) has three unknown coefficients $K, L_{\infty}$ and $t_{0}$, to be determined analytically on the basis of given experimental data (i.e. the length and age measurements of the species under analysis). A great number of methods for estimating von Bertalanffy growth parameters have been successfully implemented in practice, with their main disadvantage being the requirement for regularity of the corresponding measurements. Failure to collect and process the data on a regular basis distorts the results of the methods discussed so far except for the Gulland-and-Holt plot, and what is more, they usually provide estimates for only 2 of the 3 unknown parameters (Sparre \& Venema, 1998), with the exception of (Melnikova, 2009).

Norbert Winner and Ludwig von Brtalanffy conclusively proved in their works the existence of close similarities in the operation and control of the machines and the living organisms. It follows, therefore, that the common principles, methods and approaches developed for the analysis and control of large technical systems can be successfully modified or adapted in the research and modeling of biological objects and systems. The System theory and more specifically the development of the System identification scientific area appear to be very suitable theoretical basis for further improvement and elaboration of the object/system modeling methods and principles (Eykhoff, 1974; Genov, 2004; Hoffman \& Frankel, 2001; Soderstrom \& Stoica, 1989).

The Least Squares Method (LSM) techniques are well-known and widely applied in various scientific areas for experimental data processing and its subsequent fitting to a model (Stigler, 1981; Walter \& Pronzato, 1997). The LSM is a standard approach in regression analysis to approximate the solution of overdetermined systems, i.e., sets of equations in which the number of equations is much higher than the parameters unknown. "Least squares" means that the overall solution minimizes the sum of the squares of the residuals made in the results of every single equation. It is widely practiced in engineering for solving regulator synthesis problems and system modeling, where a variety of LSM modifications have been developed aiming at obtaining general improvement of the accuracy in parameter estimates. The LSM also takes place in modeling and parameter estimation of biological objects. Its wide applicability is generally assumed to be associated with normal distribution of input data used for analysis provided that the number of data should exceed the number of the unknown (estimated) parameters. The present article proposes a two-step procedure designed on the methods of least squares and instrumental variables for simultaneous estimation of the three unknown parameters in von Bertalanffy growth model equation - $L_{\infty}, K$ and $t_{0}$.

\section{BACKGROUND}

For the purposes of the present analysis and development of the method proposed and the corresponding algorithm, the von Bertalanffy growth equation will be re-stated as follows:

$$
L(t)=L_{\infty}-L_{\infty} e^{-K(t-t 0)}
$$


In terms of the General Theory of Automatic Control and System Identification, equation (2) is considered transition (step) function of the object/system under consideration.

Numerous methods have been developed to determine the system parameters by its step response, which prove to be ineffective in offering a solution to the von Bertalanffy growth equation. Thus, despite having the same model, the methods for determining the parameters of the transition (step) function based on experimental data valid for the technical systems are not applicable for biological objects. Hence, a new approach to solving the Growth equation was considered necessary.

In the case of fish, the growth rate measured in length is represented as:

$$
\frac{d L(t)}{d t}=L_{\infty} e^{-K\left(t-t_{0}\right)} K
$$

Equation (2) can be then rewritten:

$$
L_{\infty} e^{-k(t-t 0)}=L_{\infty}-L(t)
$$

If (4) is substituted in (3), the following shall be obtained:

$$
\frac{d L(t)}{d t}=k \cdot L_{\infty}-k \cdot L(t)
$$

Since $L_{\infty}$ is constant, the relationship between the growth rate $\frac{d L(t)}{d t}$ and the length $L(t)$ is a linear function. Accordingly, it makes it possible for the unknown parameters $L_{\infty}$ and $K$ to be easily estimated by a linear regression analysis (provided that length-at-age data (measurements) is available). It should be emphasized, however, that the accuracy of the parameter estimates is strongly dependent on the input data quality. Great accuracy of estimates can only be expected if the length and age are determined precisely. Experimental data collection and sampling are normally accompanied by added noise of different types and levels, for example as a result of inaccurate measurements. The present analysis takes into consideration random error occurrence, rather than systematic measurement error, and thereupon it is reasonable to assume that those random errors have zero mean or expectation $M=0$, dispersion $\sigma$ and are normally distributed. The a priori analysis of the problem is of major significance for further selection of mathematical (instruments) solution (procedure) to the growth equation. Given the above listed considerations and assumptions, the present paper introduces Twostep LS and IV- method based procedure for simultaneous estimation of the individual growth parameters $L_{\infty}, K$ and $t_{0}$.

\section{Growth Parameters Estimation}

Length-at-age samples in general present discrete data in relation to the parameters measured and the values $L(i \Delta t)$ are accordingly discrete, which is to mean that the continuous derivative (5) can be duly expressed as the finite difference $\frac{\Delta L}{\Delta t}$. The theory of finite difference approximations of derivatives is covered in most numerical analysis textbooks and has been widely used in scientific and engineering computations. It is generally recognized, however, that the quality of the approximation greatly depends on the regularity of the function. If the initial data is sampled from a sufficiently 
smooth function, or if the data is 'dirty' because of measurement errors, simple one-sided finite difference appears the most appropriate solution. Interpolation of data is usually related to selection of sampling time $\Delta t=$ const. Moreover, in numerical differentiation even small variations of the function can lead to major changes in its derivative. Provisions are made in the analysis under discussion for non-regular measurements of the parameters $(L(t)$ and $t$ ) and respectively $\Delta t \neq$ const. In accordance with the mean values theorem (finite increments) (Bradistilov, 1961; Bretscher, 1995), which is to be applied hereinafter for derivation of the procedure proposed:

$L\left(x+t_{i+1}\right)-L\left(x+t_{i}\right)=\Delta t \cdot L^{\prime}(x+\xi)$, where: 3/4is numerical value in the interval:

$t_{i}<\xi<t_{i+1}$. here: $\xi=\xi(x, \Delta t)$.

The calculations further are based on the following assumption:

$t_{s i}=t_{i}+\xi_{i}=t_{i}+0.5\left(t_{i+1}-t_{i}\right), \quad L^{\prime}\left(t_{s i}\right), i=\overline{1, N-1}$.

\section{Step 1: Estimation of the Asymptotic Length $L_{\infty}$ and the Parameter $K$}

It is assumed that $N=$ number of lengthmeasurements of fish - i.e. $L\left(t_{1}\right), L\left(t_{2}\right), L\left(t_{3}\right), \ldots$, $L\left(t_{N}\right)$, which correspond to ages: $t_{1}, t_{2}, \ldots, t_{N}$, the linear equation (5) then can be expressed by the following system of equations:

$L^{\prime}\left(t_{s i}\right)=k \cdot L_{\infty}-k \cdot L\left(t_{s i}\right), i=\overline{1, N-1}$

or in matrix-vector form as:

$L^{\prime}=k \cdot L_{\infty} .1-k \cdot L s$

where:

$$
\begin{aligned}
& L^{\prime}=\left[L^{\prime}\left(t_{s 1}\right), L^{\prime}\left(t_{s 2}\right) L^{\prime}\left(t_{s 3}\right), \ldots, L^{\prime}\left(t_{s, N-1}\right)\right]^{T} ; \\
& L s=\left[L\left(t_{s 1}\right), L\left(t_{s 2}\right), L\left(t_{s 3}\right), \ldots, L\left(t_{s, N-1}\right)\right]^{T} ;
\end{aligned}
$$

1 - is a vector of ones with size $N-1$.

Equation (7) can also be conveyed as:

$L^{\prime}=[1-L s] \cdot\left[\begin{array}{c}K L_{\infty} \\ K\end{array}\right]=X . b$ 
Where the elements of the vector $\mathrm{b}$ are the unknown von Bertalanffy growth parameters $\mathrm{K}$ and $\mathrm{L}_{\infty}$.

Supposing that $\hat{b}$ is the estimation of vector $b$, the error vector e should be defined and respectively taken into account when $\hat{b}$ is additionally substituted in (9) to obtain the following equation:

$$
e=L^{\prime}-X . \hat{b}
$$

The elements of the error vector e can also contain measurement errors.

The unknown vector $\hat{b}$ will be determined in accordance with the minimum norm least squares solution requirement, i.e.:

$$
J=e^{T} e=\underbrace{\min }_{\hat{b}}
$$

In compliance with the requirement for minimization of the norm (11), it follows that:

$$
\hat{b}=\left[X^{T} \cdot X\right]^{-1} X^{T} L^{\prime}=\mathrm{C} \cdot \mathrm{X}^{\mathrm{T}} \mathrm{L}^{\prime}
$$

As a result, the elements of vector $\hat{b}$ shall determine $L_{\infty}$ and $K$ :

$$
L_{\infty}=\frac{b(1)}{b(2)}, \quad K=b(2)
$$

\section{Step 2: Estimation of $t_{0}$}

Once the 2 growth parameters $L_{\infty}$ and $K$ have been calculated in the first step of the procedure being described - equation (2) can be re-stated as follows:

$$
e^{-K(t-t 0)}=1-\frac{L(t)}{L_{\infty}}=1-l(t)
$$

After taking a logarithm on both sides and carrying out some simple transformations we have:

$$
K . t_{0}=K . t+\ln |1-l(t)|
$$

If $y(t)=(\ln |1-l(t)|) / K$ is substituted in (15):

It proceeds from (16) that: 
$y(t)=t_{0}-t$

(17) is also a linear equation and for $t_{i}, i=\overline{1, N}$ a system of linear algebraic equations will take place and may be hereby presented in a matrix-vector form as:

$Y=t_{0} .1-T$

where:

$Y=\left[y\left(t_{1}\right), y\left(t_{2}\right), \ldots, y\left(t_{N}\right)\right]^{T}, T=\left[t_{1}, t_{2}, t_{3}, \ldots ., t_{N}\right]^{T}$, and $1-$ is a vector of ones with size $\mathrm{N}$.

(18) is an overdetermined system of linear equations. The unknown parameter $t_{0}$ will be established in accordance with the minimum norm least squares solution requirement, analogously to (11), once the estimation $\hat{t}_{0}$ is substituted in (18).

$\mu=(\mathrm{T}+\mathrm{Y})-\hat{\mathrm{t}}_{0} \cdot 1=\mathrm{Z}-\hat{\mathrm{t}}_{0} \cdot 1$

The unknown parameter $\hat{t}_{0}$ is to be determined through compliance with the requirement for minimization of the norm:

$G=\varepsilon^{T} \varepsilon=\min$

$=>\hat{\mathrm{t}}_{0}=\left[1^{\mathrm{T}} \cdot 1\right]^{-1} 1^{\mathrm{T}} \cdot Z=\frac{\sum_{\mathrm{i}=1}^{\mathrm{N}} \mathrm{Z}_{\mathrm{i}}}{\mathrm{N}}$

\section{Implementation of the Instrumental Variable (IV)}

IV is used when an explanatory variable of interest is correlated with the error term, in which case ordinary least squares are likely to produce biased results. A valid instrument induces changes in the explanatory variable but has no independent effect on the dependent variable, allowing a researcher to uncover the causal effect of the explanatory variable on the dependent variable. Instrumental variable methods allow for consistent estimation when the explanatory variables (covariates) are correlated with the error terms in a regression model. Such correlation may occur when changes in the dependent variable change the value of at least one of the covariates, which is subject to measurement error. Explanatory variables affected severely by one or more of these issues in a regression-based context are commonly referred to as endogenous. In such a situation, ordinary least squares produces biased and inconsistent estimates. However, if an instrument is available, consistent estimates may still be obtained. An instrument is a variable that does not itself belong to the explanatory equation but is correlated with the endogenous explanatory variables, contingent upon the value of the other covariates. Using IVs in linear models relates to two main requirements: 
The instrument must be correlated with the endogenous explanatory variables, conditional on the other covariates. If this correlation is strong, then the instrument is said to have a strong first step. A weak correlation may provide misleading inferences about parameter estimates and standard errors (Bound, Jaeger \& Baker, 1995).

The instrument cannot be correlated with the error term in the explanatory equation, conditional on the other covariates. In other words, the instrument cannot run into the same problem as the original predicting variable. If this condition is met, then the instrument is said to satisfy the exclusion restriction (Angrist \& Krueger, 2001; Bowden \& Turkington, 1984; Stock \& Trebbi, 2003).

The primary aim of IV implementation (instrumental matrix V ) in the procedure brought forward is to ensure improvement in the quality of the estimates (vector $\hat{b}$ ), more specifically in the presence of added noise (measurement error). IV has been selected to have a form identical to the structure of and in close correlation with the matrix $\mathrm{X}$ rather than with the added noise (the measurement error). It should be regarded as a special supplement to the LSM, completing and enhancing it. In general, IV derivation methodology follows the basic algorithm for the LSM.

1. Implementation of the IV for calculation of the vector $\hat{b}_{i p}$

In equation (12) used in place of $\hat{b}$ is the following expression:

$$
\hat{b}_{i p}=\left[V^{T} \cdot X\right]^{-1} V^{T} L^{\prime}
$$

where the instrumental matrix $V^{T}$ is in a form identical to the matrix $X^{T}$, and yet its elements are not the measured values $L\left(t_{s i}\right)$ but the ones forecasted by the model calculated with the (previously) estimated parameters $L_{\infty}, K$ and $t_{o}$ (as determined in Step 1. and Step 2.)

The IV is implemented in line with the following algorithm (Genov, 2004):

1. Estimate vectors $\hat{b}$ and $\hat{t}_{0}$ on the basis of the LSM;

2. Use the estimates $\hat{b}$ and $\hat{t}_{0}$ to forecast the model values $L_{\text {model }}$;

3. Use the model values $\mathrm{L}_{\text {model }}$ to form the instrumental matrices $\mathrm{V}$ and $\mathrm{V}_{1}$ and obtain the new estimates by applying the equations (21) and (22);

4. Go back to step (2);

5. The procedure is iterative and can be executed as many times as needed (usually 2 to 5 ) to secure stabilization of the estimated parameter values.

2. Implementation of the IV for calculation of the parameter $\hat{t}_{0 i p}$

The instrumental matrix $V_{1}$ should be selected in a form identical to the matrix $Z$, but in this case $y$ will be formed by implementing the model forecasted values $L_{\text {model }}$, that have been calculated using the already estimated values $\hat{b}$ and $\hat{t}_{0}$;

Upon substitution of the vector of ones in (21) with $V_{1}^{T}$, the parameter $\hat{t}_{0 i p}$ is to be calculated through the following equation: 


$$
\hat{t}_{0 i p}=\left[V_{1}^{T} \cdot 1\right]^{-1} V_{1}^{T} \cdot Z=\frac{\sum_{i=1}^{N} v_{1 i} \cdot z_{i}}{\sum_{i=1}^{N} v_{1 i}}
$$

3. Analysis of the model accuracy $L_{\text {model }}$

The unknown parameters of the model (2) having been estimated, its accuracy should be evaluated, i.e. at what extent the model data comes close to the experimental (the measured) data. To that effect, it is necessary for the calculated model values to be compared to the experimental data. The smaller the difference is, the greater the model accuracy. Higher deviations of the model estimates $L_{\text {model }}$ from the measurement data $L(t)$ are indications for incorrect model structure and respectively, low quality of the parameter estimates or inaccurately defined measurement error impact (incorrect measurement in the length or age of fish). As a measure of the model accuracy it is appropriate to adopt the error variance (or the standard deviation): $S_{\text {emodel }}^{2}=\frac{1}{N-1} \sum_{i=1}^{N}\left(L_{i}-L_{\text {model }, i}\right)^{2}$, or the model relative error: $S_{\text {otn_model }}=S_{\text {emodel }} / S_{L}$.

\section{MATLAB Program for the Realization of the Proposed Two- step LS and IV- Method Based Procedure for Simultaneous Estimation of the Individual Growth Parameters $L_{\infty}, K$ and $t_{0}$}

For the purposes of the practical implementation of the procedure described and its satisfactory performance a MATLAB-environment script has been developed - provided for reference in Appendix A.

\section{MAIN FOCUS OF THE ARTICLE}

\section{Performance and Operational Examination of the Two-step Procedure for Simultaneous Estimation of the von Bertallanfy Growth Parameters $L_{\infty}, K$ and $t_{0}$}

For the objectives of the present research, the subsequent in-depth analysis and concrete proof of the workability of the improved Two-step LS and IV- method based procedure for simultaneous estimation of the individual growth parameters $L_{\infty}, \mathrm{K}$ and $t_{0}$ put forward by the paper, the results obtained will be compared with the results produced by other widely used methods for estimating von Bertallanfy growth parameters. Simultaneously with the execution of the procedure under discussion and with the provision of equal conditions applied consecutively are two methods for estimating the above-mentioned parameters $L_{\infty}, K$ and $t_{0} . K$ and $L_{\infty}$ are determined first by applying the Gulland-and-Holt method and then the calculated asymptotic size value was used to estimate the $t_{0}$ by the von Bertalanffy method.

Once the goal was set, a specific MATLAB-based program was developed for consecutive execution of Gulland-and-Holt method for estimation of $K$ and $L_{\infty}$ and the von Bertalanffy method for estimation of $t_{0}$, which is fed along with the asymptotic length value $L_{\infty}$ previously estimated using the former method. The content of the program script is presented for reference in Appendix B. 


\section{Simulation Data}

Input data used for the simulation series:

$\mathrm{t}_{0}=0.087 ; \mathrm{K}=0.78 ; \mathrm{L}_{\infty}=50.00 \mathrm{~cm}$, the data is simulated to present non-regular measurements $(\Delta t \neq$ const $)$ in the interval $0.1-4.35$ years, by using the von Bertalanffy model: $L(t)=L_{\infty}\left[1-\exp \left(-K\left(t-t_{0}\right)\right)\right]$ in MATLAB program environment with the following program script:

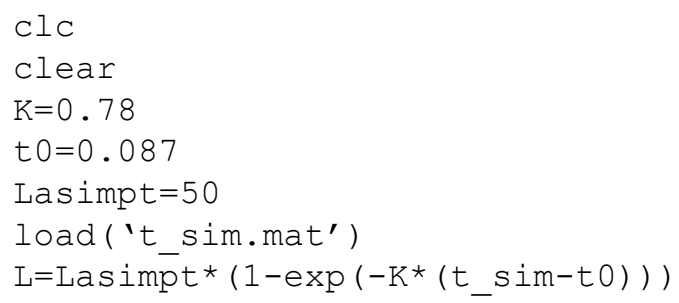

Along with the study into the accuracy of the growth parameter estimates obtained by the methods described so far, another study was carried out into the probable way the measurements might affect the estimates and the level of resistance to the added noise of the methods themselves (Fuller, W. A. (1987)). Provisions were made for simulated cases of added measurement error through implementation of random normally distributed error, which is considered either incorrect measurement of $\mathrm{L}$ or incorrect determination of the corresponding ages. The values $L=L_{-}$sim and $t=t_{-}$sim simulated by the von Bertalanffy growth model were completed with a normally distributed error, generated by using the following script:

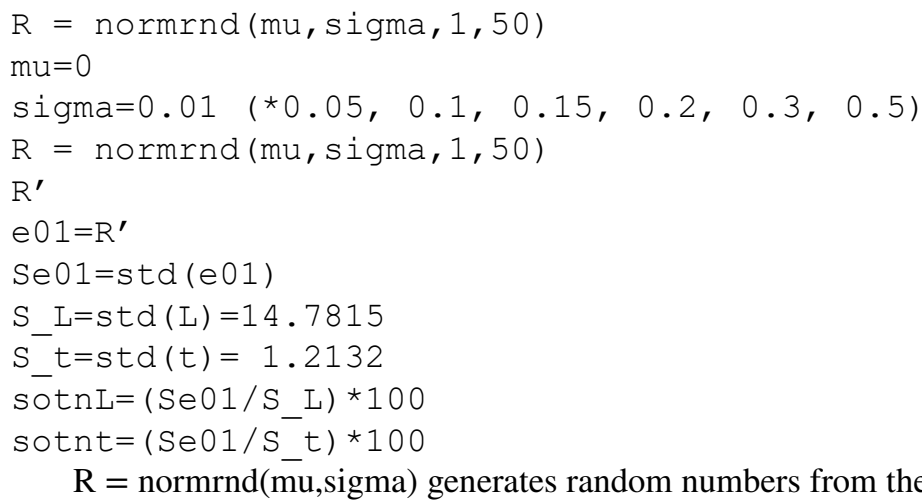
parameter mu and standard deviation parameter sigma. Mu and sigm multidimensional arrays that have the same size, which is also the size of $\mathrm{R}$. A scalar input for mu or sigma is expanded to a constant array with the same dimensions as the other input.

In order to address the noise levels to a certain percentage, added measurement error is calculated:

- The standard deviation of the input data is to be determined by:

$S_{L}=\operatorname{Std}(L)=14.7815$ and: $S_{t}=S t d(t)=1.2132$;

- Signal-to-noise ratio: $S_{o t n L}=\frac{S_{e}}{S_{L}} 100(\%)$ and $S_{o t n t}=\frac{S_{e}}{S_{t}} 100(\%)$; 
The generated noise (added measurement errors) has the following characteristics:

$e_{01}$ - standard deviation $S_{e_{01}}=0.0103$; signal-to-noise ratio $S_{\text {otnL }}=0.0730 \%$ or measurement error $\approx 0.07 \%$ and $S_{\text {otnt }}=0.8897$ or measurement error $\approx 1 \%$

$e_{05}$ - standard deviation $S_{e_{05}}=0.0557$; signal-to-noise ratio $S_{\text {otnL }}=0.3769$ or measurement error $\approx 0.4 \%$ and $S_{\text {otnt }}=4.5918$ or measurement error $\approx 5 \%$

$e_{1}$-standard deviation $S_{e_{1}}=0.1036$; signal-to-noise ratio $S_{o t n L}=0.7006$ or measurement error $\approx 0.7 \%$ and $S_{\text {otnt }}=8.5356$ or measurement error $\approx 10 \%$

$e_{15}$ - standard deviation $S_{e_{15}}=0.1526$; signal-to-noise ratio $S_{\text {otnL }}=1.0326$ or measurement error $\approx 1 \%$

$e_{2}$ - standard deviation $S_{e_{2}}=0.1946$; signal-to-noise ratio $S_{o t n L}=1.3164$.or measurement error $\approx 1,5 \%$

$e_{3}$ - standard deviation $S_{e_{3}}=0.3364$; signal-to-noise ratio $S_{o t n L}=2.2758$ or measurement error $\approx 2 \%$

$e_{5}$ - standard deviation $S_{e_{5}}=0.5141$; signal-to-noise ratio: $S_{\text {otnL }}=3.4782$ or measurement error $\approx 3.5 \%$

$e_{6}$ - standard deviation $S_{e_{6}}=0.9149$; signal-to-noise ratio: $S_{\text {otnL }}=6.1897$ or measurement error $\approx 6 \%$

Histograms of the generated measurement errors (noise) are presented in Figure 1, Figure 2, Figure 3, Figure 4, Figure 5, Figure 6, Figure 7 and Figure 8.

The input data used for obtaining estimates of the growth parameters by the methods listed below:

- The Two-step LS and IV method-based procedure for simultaneous estimation of the individual growth parameters $L_{\infty}, K$ and $t_{0}$ advanced by the present paper

- The Gulland-and-Holt method for estimation of $L_{\infty}$ and $K$

- The von Bertalanffy method for estimation of $t_{0}$ as presented in Table 1

\section{SIMULATION RESULTS}

The results rendered by the execution of the above listed procedure and methods are presented in Table 2 and figures 9 to 11 :

\section{CONCLUSION}

Advanced in the article is a two-step procedure (algorithm), based on the LSM and IV implementation for simultaneous estimation of the three unknown parameters in von Bertalanffy growth equation. Specific software in MATLAB programming environment has additionally been developed for the purposes of the present analysis.

To assess the forecasting quality and accuracy of the approximating model use is made of the variance (standard deviation) of the model error - i.e. the difference between the measured (simulated) values and those forecasted (estimated) by the model. 
Figure 1. Histogram (distribution shape) of the simulated measurement error $\mathrm{e}_{01}$ (left)

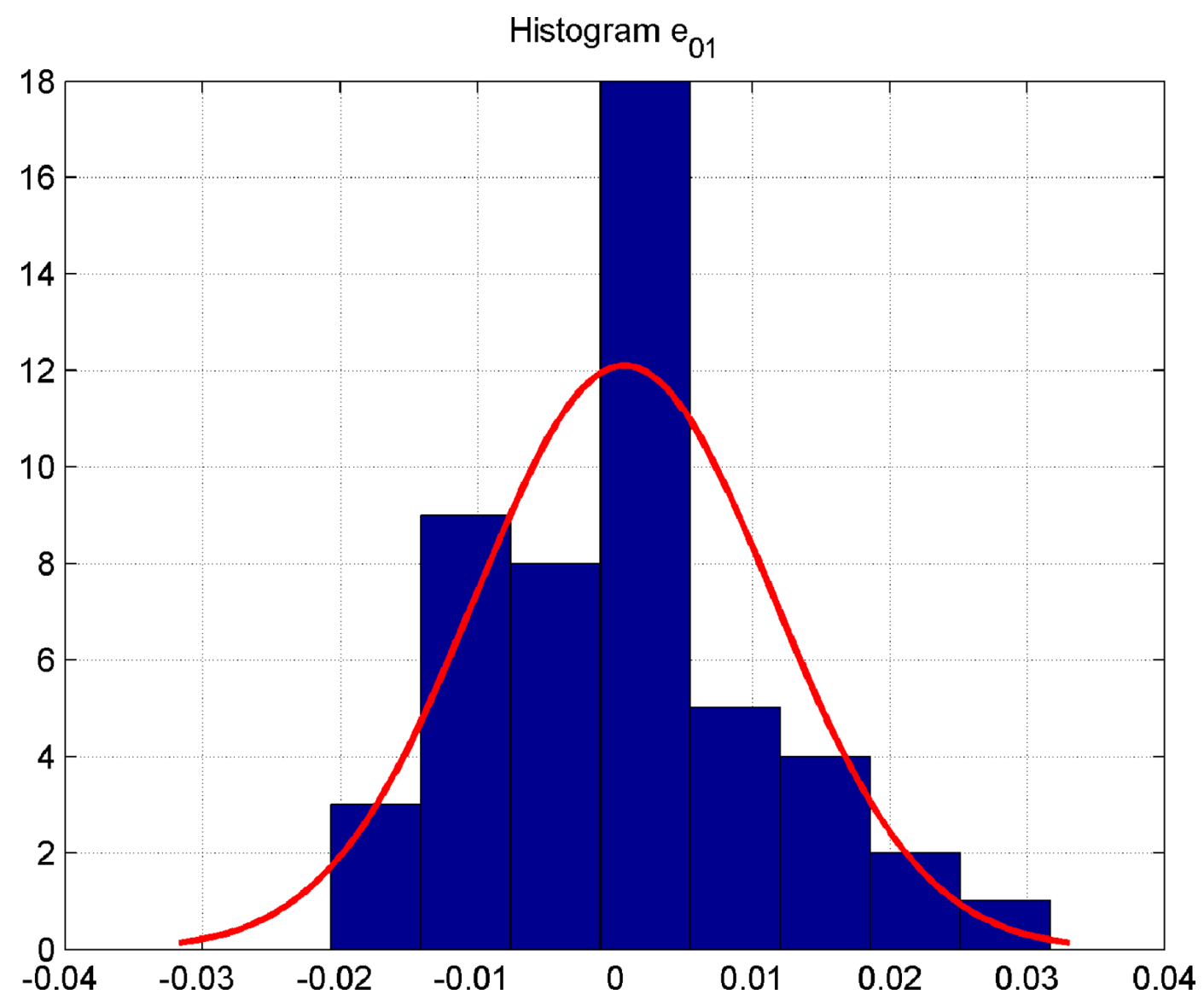

Research studies have been carried out with extra levels of added noise (input of a measurement error in the length or age determination of the given biological object) with the noise burden being normally distributed and generated by the normrnd function with a mathematical expectation of $M=0$ and a standard deviation $\tilde{\mathrm{A}}=\mathrm{S}$ from 0.01 to 0.5 . The simulated measurement error is $0.07 \%$ to $3.5 \%$ for $L$ and $1 \%$ and $5 \%$ for $t$.

The results of the conducted experiments as to the two-step LS and IV method-based procedure for simultaneous estimation of von Bertalanffy growth parameters: $L_{\infty}, K$ and $t_{0}$ have been compared to the results obtained by the Gulland-and-Holt method for estimation of $L_{\infty}$ and $K$ and subsequent execution of von Bertalanffy method for estimation of $t_{0}$. To achieve the stated objective, developed has been a MATLAB-based program for consecutive implementation of the selected two methods.

The estimates of the growth parameters produced by the proposed procedure for simultaneous estimation of $L_{\infty}, K$ and $t_{0}$ in the case of non-dirty (non-noisy) data were characterized with a very high degree of accuracy and the two methods were found to be comparatively noise-resistant to added measurement error of $\mathrm{L}$ and with values of the added error being up to $3.5 \%$, they tend to yield estimates with a relative accuracy of 1 to $6 \%$, thus, identifying $t_{0}$ as the most noise-sensitive parameter.

The results show conclusively that in the presence of higher levels of added noise the estimates of the growth parameters improved significantly due to the applied instrumental variable, which makes the hitherto described two-step LS and IV method-based procedure for simultaneous estimation 
Figure 2. Histogram (distribution shape) of the simulated measurement error $\mathrm{e}_{05}$ (right)

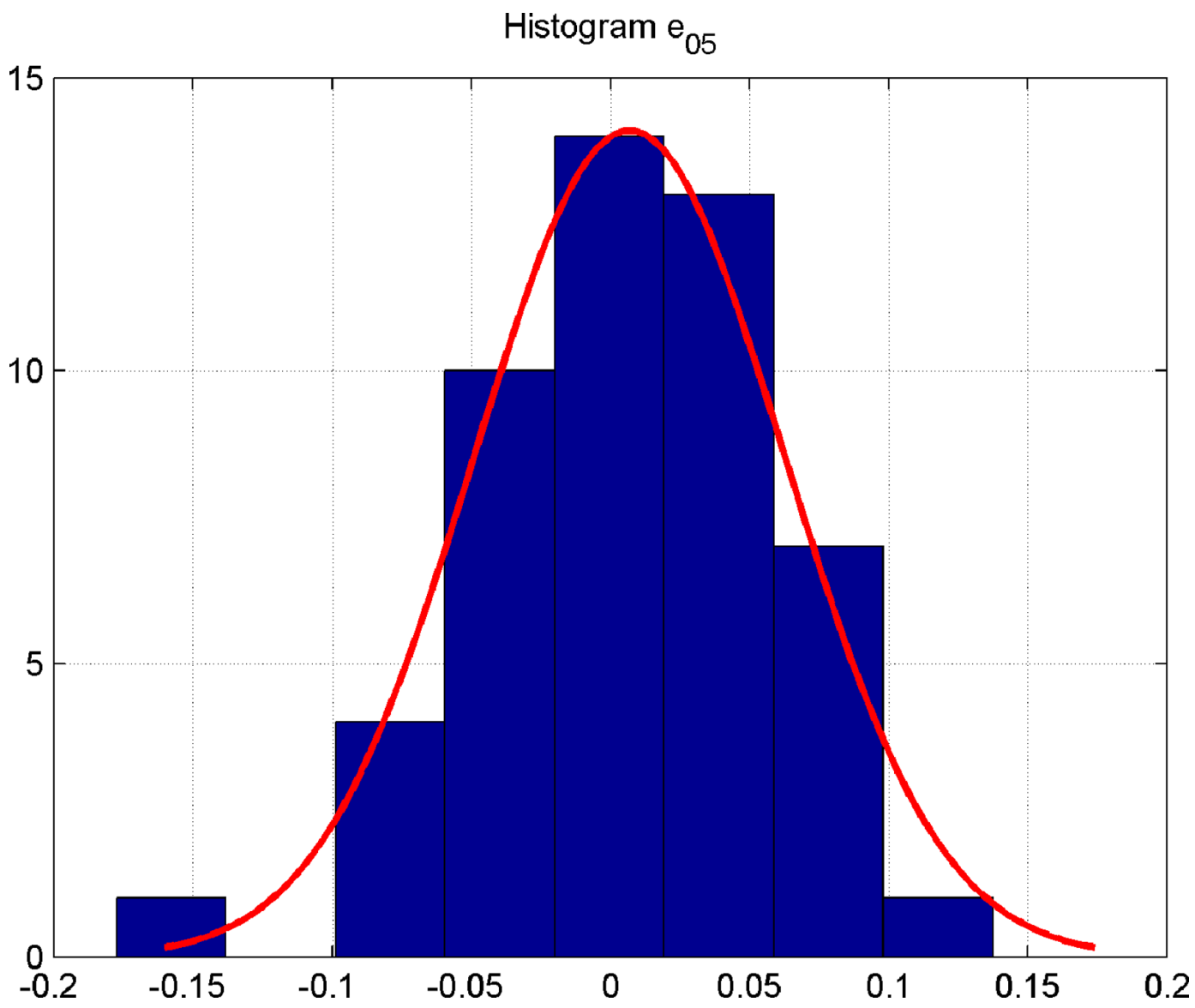

of von Bertalanffy growth parameters: $\mathrm{L}_{\infty}, \mathrm{K}$ and $\mathrm{t}_{0}$ extremely appropriate with lower quality of input data (if there are length measurement errors of around 3-4\%).

The two surveyed approaches to estimating the growth parameters proved to be particularly sensitive in terms of accuracy upon application of a measurement error in estimating $t_{0}$, and made it necessary for the issue of age determination to be addressed with utmost attention.

The analyzed two-step LS and IV method-based procedure for simultaneous estimation of von Bertalanffy growth parameters: $L_{\infty}, K$ and $t_{0}$ is applicable not only to data measurements of parameters $L$ and $t(\Delta t=$ const $)$ at regular intervals, but also to non-regular measurements of the these parameters $(\Delta t \neq$ const $)$, provided that the number of data is greater than that of the parameters under estimation. It should be borne in mind that the qualities of the instrumental variable as regards the noise resistance of the estimates are most pronounced with substantial amount of input data and the number of iterations ( $\mathrm{klu}$ ) could be increased until the right stabilization of the estimates has been secured. 
Figure 3. Histogram (distribution shape) of the simulated measurement error $e_{1}$ (left)

Histogram $\mathbf{e}_{1}$

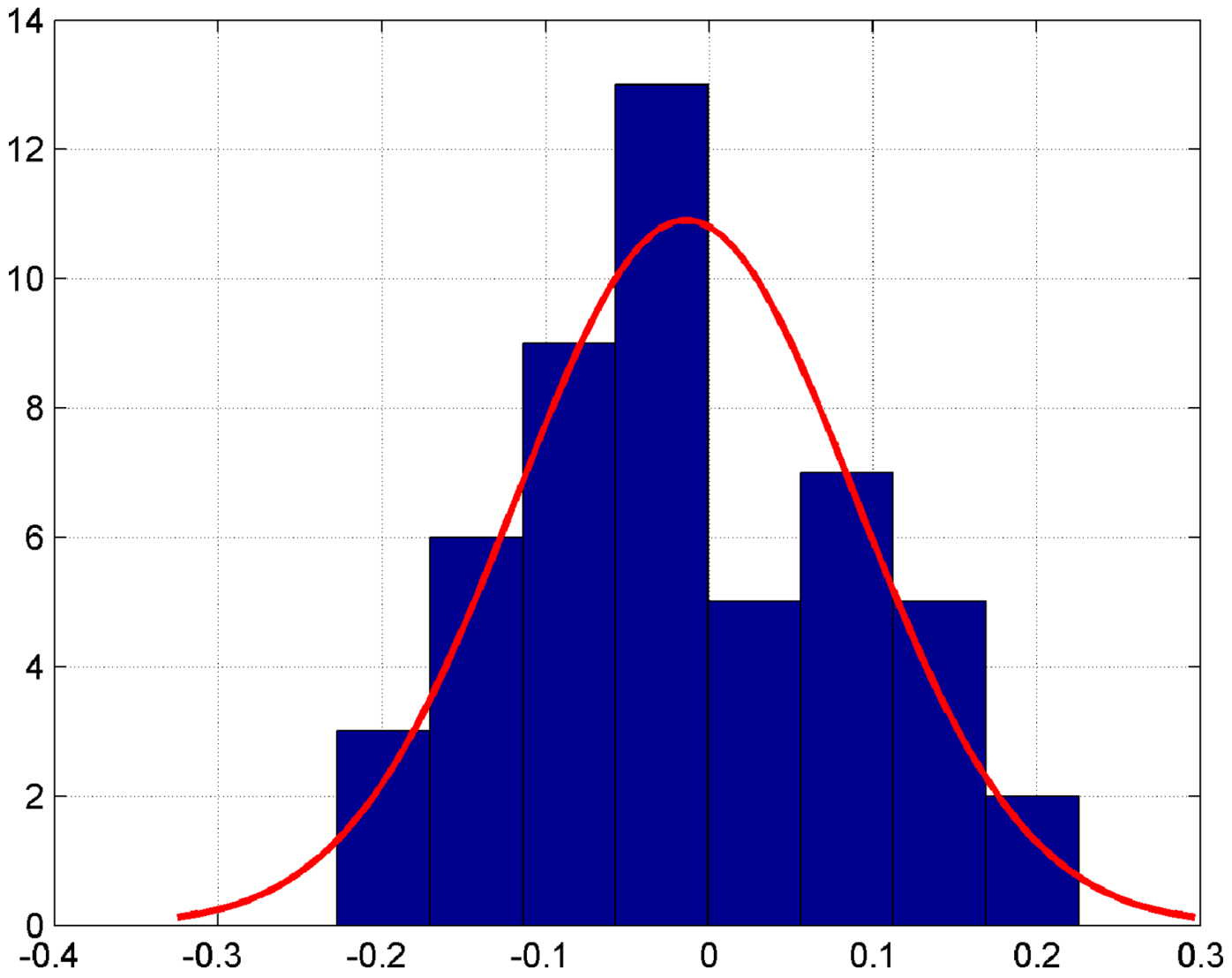


International Journal of Agricultural and Environmental Information Systems Volume 10 • Issue 2 • April-June 2019

Figure 4. Histogram (distribution shape) of the simulated measurement error $\mathrm{e}_{15}$ (right)

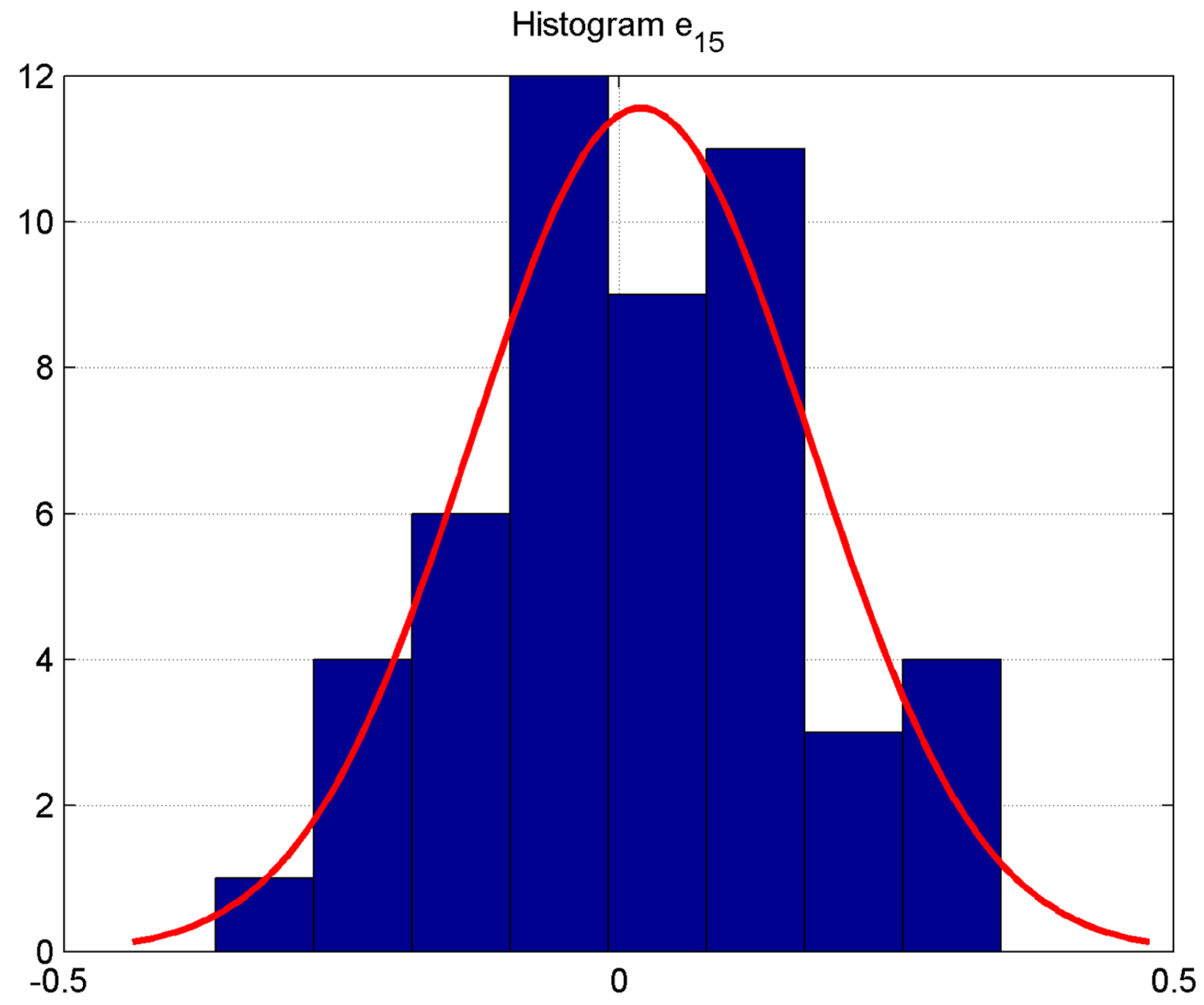


Figure 5. Histogram (distribution shape) of the simulated measurement error $\mathrm{e}_{2}$ (left)

Histogram $\mathrm{e}_{2}$

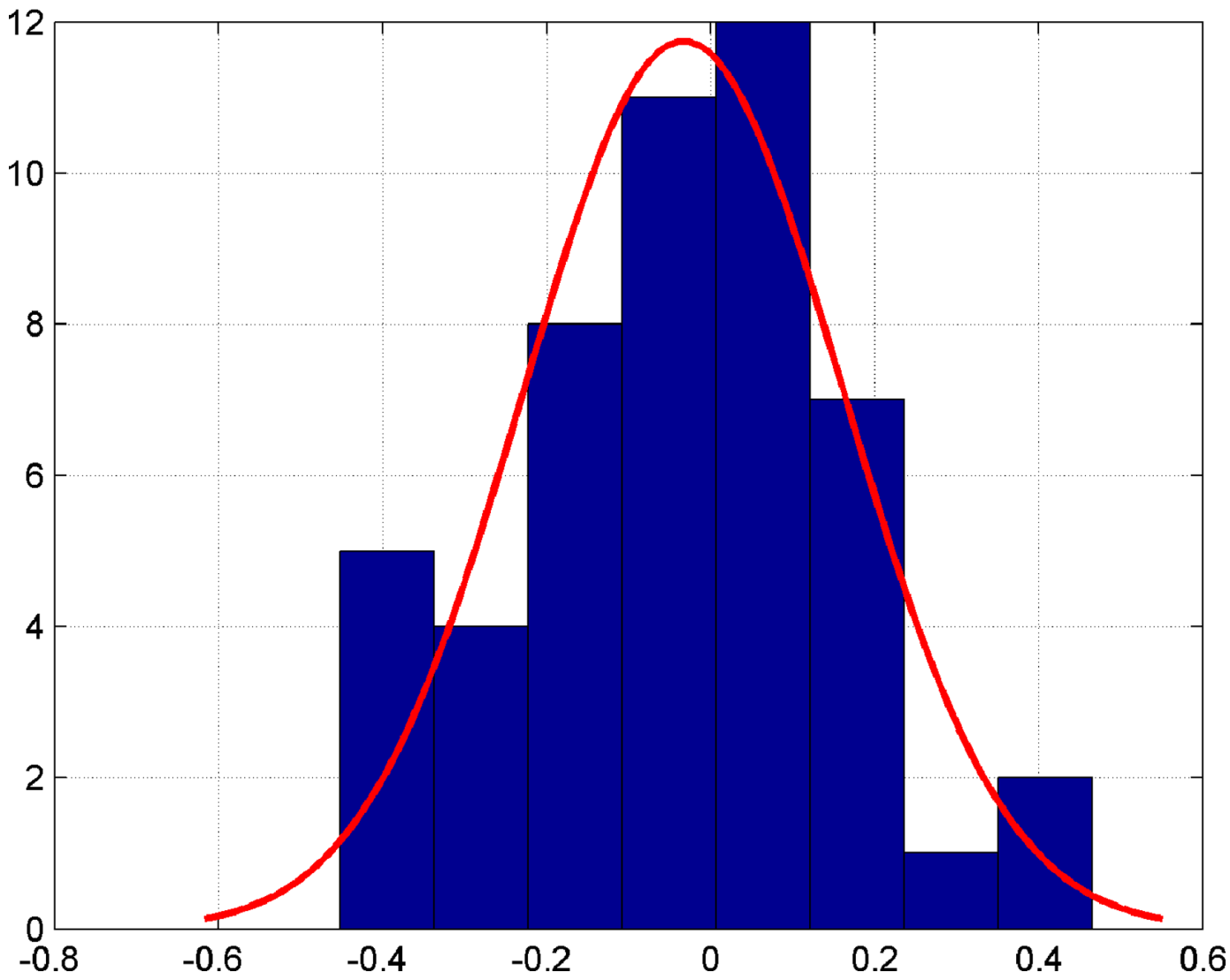


International Journal of Agricultural and Environmental Information Systems

Volume 10 •Issue 2 • April-June 2019

Figure 6. Histogram (distribution shape) of the simulated measurement error $\mathrm{e}_{3}$ (right)

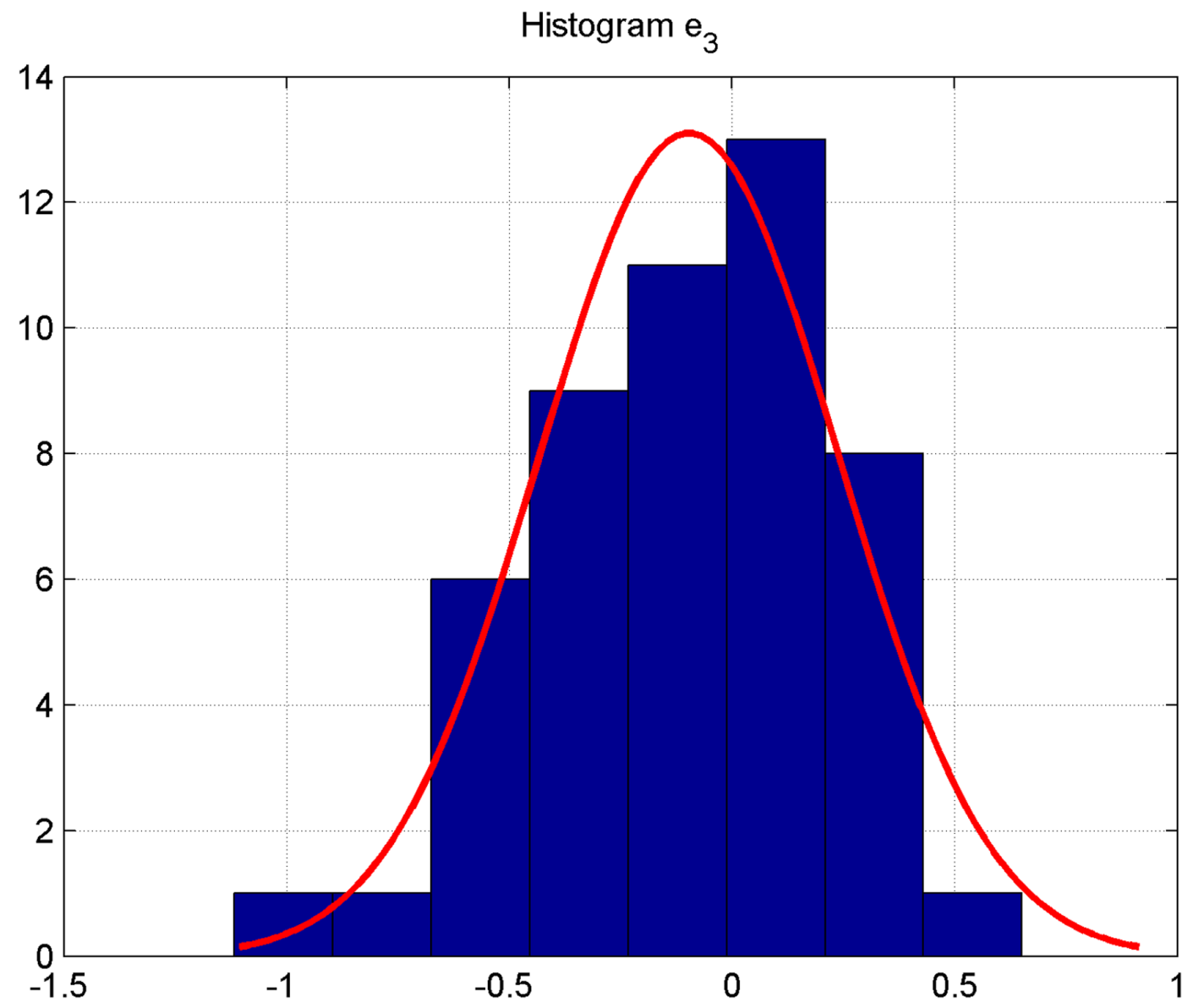


Figure 7. Histogram (distribution shape) of the simulated measurement error $\mathrm{e}_{5}$ (left)

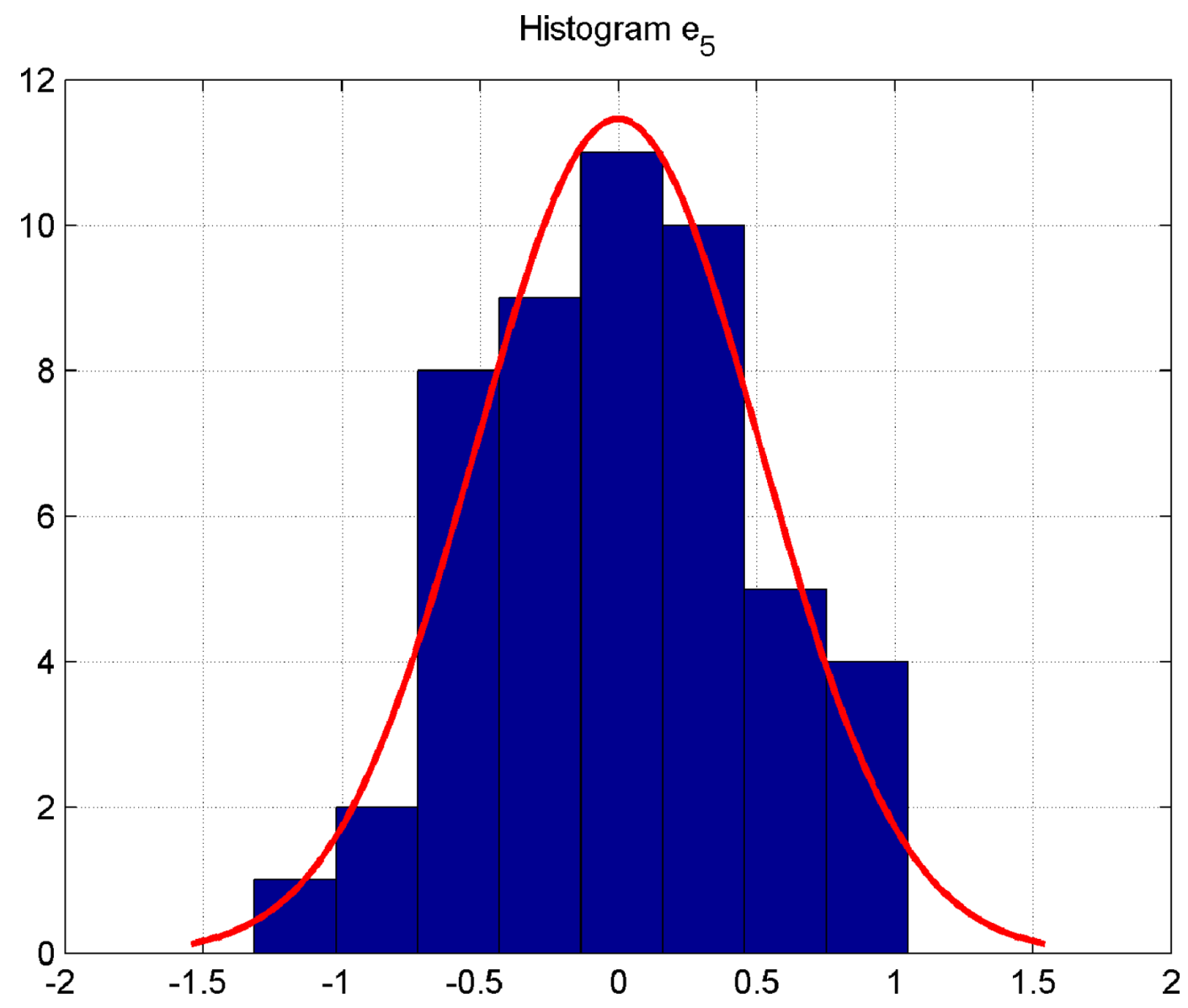


International Journal of Agricultural and Environmental Information Systems

Volume 10 •Issue 2 • April-June 2019

Figure 8. Histogram (distribution shape) of the simulated measurement error $\mathrm{e}_{6}$ (right)

Histogram $\mathrm{e}_{6}$

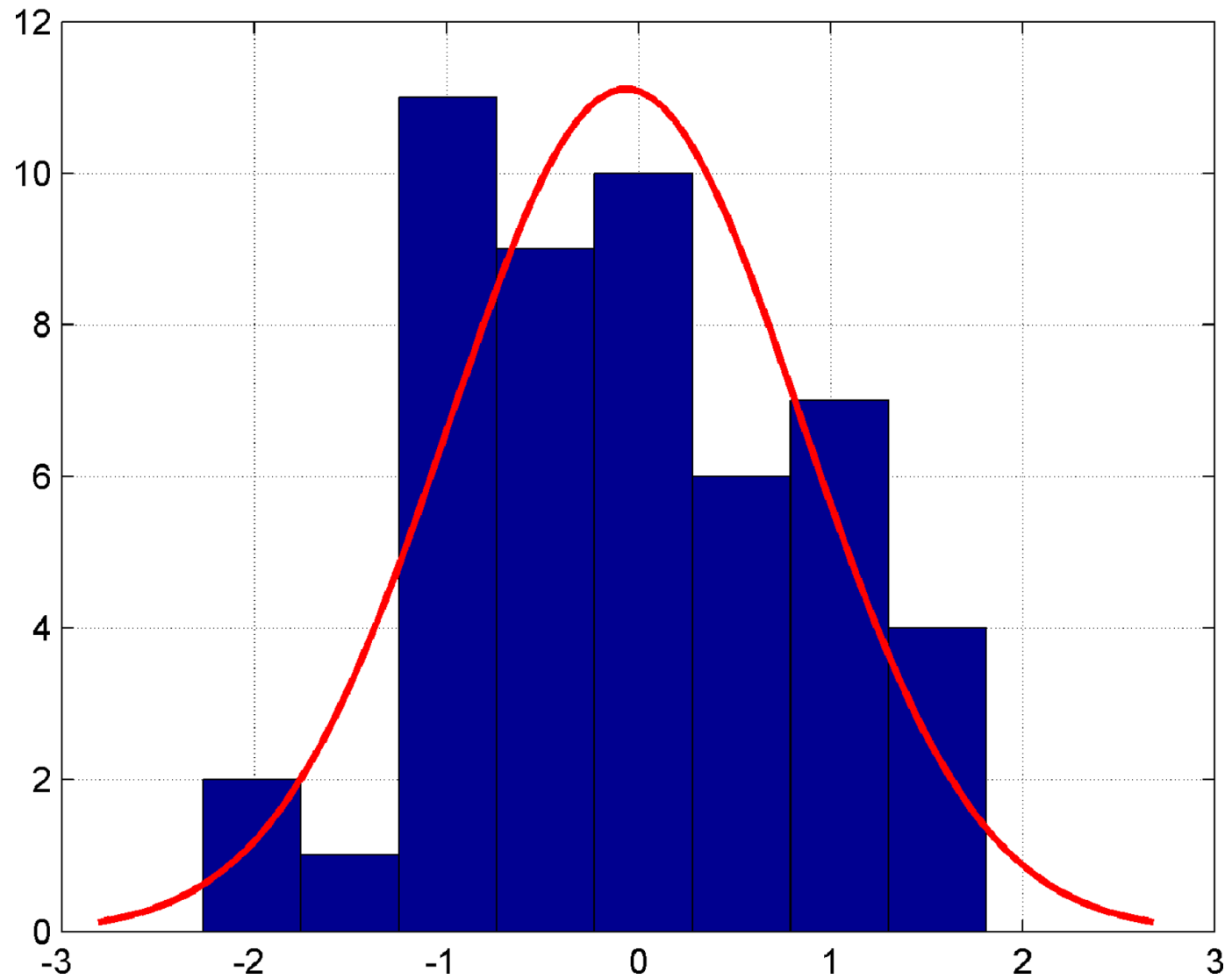


Table 1. Simulated data used to examine the operational workability and estimating accuracy of the calculation procedures and methods and noise levels of $t$ and $\mathrm{L}$

\begin{tabular}{|c|c|c|c|c|c|c|c|c|c|}
\hline$t=t_{\text {sim }}$ & $\mathbf{L}=\mathbf{L}_{\text {sim }}$ & $\mathrm{e}_{01}$ & $\mathrm{e}_{05}$ & $e_{1}$ & $\mathrm{e}_{15}^{*}$ & $\mathrm{e}_{2}^{*}$ & $\mathbf{e}_{3}{ }^{*}$ & $\mathrm{e}_{5}^{*}$ & $e_{6}^{*}$ \\
\hline 0.1000 & 0.5044 & -0.0070 & -0.0108 & -0.1701 & -0.0403 & 0.1269 & -0.7347 & 0.0121 & -0.6466 \\
\hline 0.1400 & 2.0249 & 0.0039 & 0.1376 & 0.0791 & 0.2156 & 0.0639 & -0.5375 & 1.0478 & 1.4042 \\
\hline 0.1700 & 3.1344 & -0.0083 & -0.0708 & 0.1387 & -0.0809 & -0.0696 & -0.2500 & -0.0942 & -0.7391 \\
\hline 0.2100 & 4.5741 & -0.0125 & -0.0122 & -0.0183 & -0.0645 & -0.0007 & -0.2621 & -0.3221 & -0.2809 \\
\hline 0.2600 & 6.3116 & -0.0140 & 0.0020 & -0.0908 & 0.0976 & 0.1236 & -0.4801 & -0.5039 & 0.0681 \\
\hline 0.2900 & 7.3220 & 0.0028 & -0.0687 & 0.1227 & -0.1353 & 0.4657 & -0.2035 & -0.9055 & -1.4299 \\
\hline 0.3300 & 8.6330 & 0.0195 & 0.0540 & -0.0511 & 0.1413 & 0.1773 & -0.2794 & -0.2447 & -0.8577 \\
\hline 0.3900 & 10.5244 & 0.0020 & 0.0372 & -0.0818 & -0.2525 & -0.0488 & 0.3301 & 0.1507 & -1.0996 \\
\hline 0.4100 & 11.1354 & 0.0049 & 0.0771 & -0.1262 & -0.3631 & -0.3946 & -0.1830 & -0.4920 & -2.2647 \\
\hline 0.4500 & 12.3293 & 0.0014 & 0.0344 & -0.1468 & -0.0238 & 0.0498 & -0.3828 & 0.1267 & 1.8131 \\
\hline 0.5100 & 14.0517 & 0.0023 & -0.1775 & 0.0276 & 0.0493 & 0.0522 & -0.1249 & 0.3940 & -0.6208 \\
\hline 0.5300 & 14.6081 & 0.0237 & -0.0484 & -0.0332 & 0.1913 & -0.1583 & -0.2055 & -0.2966 & 0.7141 \\
\hline 0.5700 & 15.6953 & -0.0078 & 0.0156 & 0.0788 & 0.0180 & 0.2019 & 0.0786 & 0.2461 & 0.0146 \\
\hline 0.6400 & 17.5181 & -0.0016 & -0.0408 & -0.0057 & 0.1432 & 0.0782 & -0.0694 & 0.2827 & -0.8450 \\
\hline 0.6900 & 18.7605 & -0.0067 & 0.0142 & -0.0210 & 0.0361 & 0.1174 & -0.4457 & -0.0214 & -0.0343 \\
\hline 0.7200 & 19.4830 & 0.0071 & -0.0033 & -0.1721 & 0.1019 & -0.0972 & 0.1741 & 0.9782 & 0.3440 \\
\hline 0.7800 & 20.8783 & 0.0026 & -0.0274 & -0.0775 & -0.1453 & -0.1368 & -0.1092 & -0.6361 & -0.9714 \\
\hline 0.8500 & 22.4257 & -0.0033 & 0.0034 & 0.0104 & -0.0115 & -0.0935 & -0.1800 & 0.1375 & 1.0184 \\
\hline 0.8900 & 23.2728 & 0.0074 & 0.0256 & -0.0428 & -0.0897 & -0.2221 & -0.2555 & -0.4634 & 1.0173 \\
\hline 0.9300 & 24.0938 & -0.0101 & 0.0572 & 0.1275 & -0.0355 & -0.1045 & 0.2382 & -0.3609 & -0.0828 \\
\hline 1.1000 & 27.3109 & 0.0012 & -0.0447 & -0.1831 & 0.3445 & 0.1454 & -0.0327 & 0.2205 & 0.5222 \\
\hline 1.1600 & 28.3483 & 0.0020 & -0.0286 & -0.0181 & -0.2106 & -0.4511 & -0.4658 & 0.5464 & -1.0358 \\
\hline 1.2100 & 29.1765 & 0.0157 & -0.0360 & 0.0115 & 0.0051 & -0.3544 & 0.6512 & 0.0851 & 0.4861 \\
\hline 1.2600 & 29.9730 & 0.0054 & 0.0424 & -0.0722 & -0.0481 & 0.0053 & -0.1025 & 0.6524 & -0.1091 \\
\hline 1.2900 & 30.4362 & 0.0003 & 0.0786 & 0.0112 & 0.0569 & 0.0657 & 0.0813 & -0.4651 & 1.3672 \\
\hline 1.3200 & 30.8886 & -0.0134 & 0.0528 & 0.1661 & 0.2689 & -0.1898 & 0.1064 & 0.1691 & -0.8135 \\
\hline 1.4000 & 32.0447 & -0.0147 & 0.0671 & -0.1050 & 0.1368 & 0.1073 & -0.3920 & -0.2654 & 1.7098 \\
\hline 1.4700 & 32.9988 & 0.0059 & 0.0125 & -0.0351 & -0.0985 & 0.2518 & 0.3663 & -0.3787 & 0.0171 \\
\hline 1.5300 & 33.7761 & -0.0070 & -0.0401 & -0.1419 & -0.2151 & 0.1367 & 0.0753 & -1.3158 & 0.3787 \\
\hline 1.6500 & 35.2258 & -0.0024 & 0.0340 & 0.0728 & 0.1272 & -0.3127 & 0.0431 & 0.3801 & 1.2321 \\
\hline 1.7200 & 36.0108 & -0.0087 & 0.0863 & -0.1206 & -0.1488 & -0.1461 & 0.4052 & 0.9374 & 0.9375 \\
\hline 1.8500 & 37.3598 & 0.0018 & 0.0253 & -0.0858 & -0.1073 & -0.1708 & 0.0477 & 0.2498 & -0.7445 \\
\hline 1.9200 & 38.0314 & 0.0023 & 0.0465 & 0.1423 & 0.0698 & -0.0678 & -0.5167 & 0.6882 & -0.7780 \\
\hline 2.1000 & 39.5992 & 0.0174 & -0.0772 & 0.0823 & -0.2284 & -0.1343 & -0.0048 & 0.0295 & -0.7776 \\
\hline 2.1700 & 40.1519 & 0.0182 & 0.0286 & -0.0756 & 0.2334 & -0.1683 & 0.3501 & -0.6152 & 0.9135 \\
\hline 2.3200 & 41.2392 & 0.0106 & -0.0077 & 0.1017 & 0.0154 & 0.3820 & 0.3638 & -0.4362 & -0.1153 \\
\hline 2.4500 & 42.0840 & 0.0108 & 0.0277 & -0.0659 & -0.0351 & -0.0666 & 0.0794 & -0.4013 & -0.3780 \\
\hline 2.5200 & 42.5046 & -0.0117 & 0.0638 & 0.0106 & 0.1212 & -0.0305 & -0.5080 & 0.5512 & 0.0701 \\
\hline 2.6400 & 43.1744 & 0.0128 & -0.0320 & 0.1834 & 0.1209 & 0.0573 & 0.3289 & -0.2402 & 0.8804 \\
\hline 2.7800 & 43.8805 & -0.0094 & 0.0753 & 0.2257 & -0.0374 & -0.3464 & -0.0172 & 0.0457 & -0.1351 \\
\hline 2.8600 & 44.2507 & -0.0160 & -0.0472 & -0.0443 & 0.1653 & -0.2283 & 0.0576 & -0.6666 & 0.2629 \\
\hline 2.9700 & 44.7234 & 0.0317 & 0.0020 & -0.0030 & 0.0551 & 0.0940 & 0.3844 & -0.0915 & -1.9681 \\
\hline 3.0100 & 44.8855 & 0.0032 & 0.0153 & 0.1039 & 0.1650 & 0.0703 & 0.1208 & -0.8129 & -0.5674 \\
\hline 3.1500 & 45.4146 & -0.0066 & 0.0533 & 0.0608 & 0.0395 & -0.2322 & -1.1167 & -0.3512 & -0.9066 \\
\hline 3.2100 & 45.6242 & 0.0001 & -0.0162 & -0.0233 & -0.0287 & 0.2251 & -0.4991 & 0.3797 & -0.5433 \\
\hline 3.4400 & 46.3429 & 0.0007 & -0.0468 & -0.0209 & 0.2623 & -0.0133 & -0.2484 & 0.8548 & -0.6477 \\
\hline 3.9300 & 47.5045 & -0.0207 & 0.0708 & -0.0784 & -0.1175 & 0.0283 & -0.3874 & 0.2427 & -0.3588 \\
\hline 4.1500 & 47.8980 & -0.0072 & 0.0181 & -0.2272 & 0.0950 & 0.0219 & 0.0460 & 0.0486 & -0.4176 \\
\hline 4.2000 & 47.9784 & 0.0053 & -0.0957 & -0.0157 & 0.3034 & -0.1100 & 0.0456 & 0.5366 & 0.5591 \\
\hline 4.3500 & 48.2016 & 0.0004 & 0.0160 & -0.1198 & -0.0620 & -0.3663 & -0.2079 & 0.3640 & 1.2207 \\
\hline
\end{tabular}

*the noise levels $\mathrm{e}_{15}, \mathrm{e}_{2}, \mathrm{e}_{3}$ and $\mathrm{e}_{5}$ are added to $L(t)$ measurements only, considering the fact that in relation to $t$ this appear to be approximately $10 \%$ error in age determination - in this case it is recommended age determination by using analytical methods or repetition of the experiment. 


\section{Table 2. Simulation results $L \infty, K$ and to}

Two-steps procedure for simultaneous estimation of the individual growth parameters $L_{\infty}, K$ and $t_{0}$, based on the LS and IV methods

(proposed in the present material)
Consecutive execution of:

1. Gulland-Holt method for estimation of $K$ and $L_{\infty}$ 2. Estimation of $t_{0}$ by the von Bertallanfy model (uses the calculated value for $L_{\infty}$ delivered upon the execution of the preceding method)

Model values: $t_{0}=0.087 ;$ yearsK $==0.78 ; L_{\infty} 50.00 \mathrm{~cm}$

1. Simulation data $t_{\text {sim }}$ и $L_{\text {sim }}$ - graphical representation is depicted in Figure 9

1.1 After the execution of the LSM

$L_{\infty}=49.9925 ; K=0.7801 ; t_{0}=0.0863$

1.2 After the implementation of the IV

$L_{\infty}=49.9925 ; \boldsymbol{K}=0.7801 ; \boldsymbol{t}_{01}=0.0863$

$S_{\text {otn_model }}=\frac{S_{e}}{S_{L}}=7.1075 * 10^{-4} \approx 0$
1.1. Gulland-Holt method for estimation of $K$ and $L_{\infty}$

$L_{\infty}=49.9925 ; K=0.7801$

1.2. Von Bertalanffy method for estimation of $t_{0}$

$\left(L_{\infty}=49.9925\right)$

$t_{0}=0.0876$

2. Simulation data: $t_{\text {sim }}$ and $\boldsymbol{L}_{01}=\boldsymbol{L}_{\text {sim }}+\boldsymbol{e}_{01}$

\subsection{After the execution of the LSM}

$\boldsymbol{L}_{\infty}=49.9380 ; \boldsymbol{K}=0.7823 ; \boldsymbol{t}_{0}=0.0845$

2.2 After the implementation of the IV

$\boldsymbol{L}_{\infty}=49.9380 ; \boldsymbol{K}=0.7823 ; \boldsymbol{t}_{0}=0.0845$

$S_{\text {otn_model }}=\frac{S_{e}}{S_{L}}=0.0031=0.31 \%$
2.1. Gulland-Holt method for estimation of $K$ and $L_{\infty}$ $\boldsymbol{L}_{\infty}=49.9381 ; \boldsymbol{K}=0.7823$

2.2. Von Bertalanffy method for estimation of $t_{0}$ $\left(L_{\infty}=49.9381\right)$

$\boldsymbol{t}_{0}=0.0914$

3. Simulation data: $\boldsymbol{t}_{\text {sim }}$ and $\boldsymbol{L}_{05}=\boldsymbol{L}_{\text {sim }}+\boldsymbol{e}_{05}$ graphical representation is depicted in Figure 10

3.1 After the execution of the LSM

$\boldsymbol{L}_{\infty}=49.8952 ; \boldsymbol{K}=0.7884 ; \boldsymbol{t}_{0}=0.0907$ 3.2 After the implementation of the IV

$\boldsymbol{L}_{\infty}=49.8952 ; \boldsymbol{K}=0.7884 ; \boldsymbol{t}_{0}=0.0907$

$S_{\text {otn_model }}=\frac{S_{\text {emodel }}}{S_{L}}=0.0052=0.52 \%$
3.1. Gulland-Holt method for estimation of $K$ and $L_{\infty}$ $\boldsymbol{L}_{\infty}=49.8930 ; \boldsymbol{K}=0.7885$

3.2. Von Bertalanffy method for estimation of $t_{0}$

$\left(L_{\infty}=49.8930\right)$

$t_{0}=0.0944$

4. Simulation data: $\boldsymbol{t}_{\text {sim }}$ and $\boldsymbol{L}_{1}=\boldsymbol{L}_{\text {sim }}+\boldsymbol{e}_{1}$ 


\begin{tabular}{|c|c|}
\hline $\begin{array}{l}4.1 \text { After the execution of the LSM } \\
\boldsymbol{L}_{\infty}=50.1409 ; \boldsymbol{K}=0.7850 ; \boldsymbol{t}_{0}=0.1158 \\
4.2 \text { After the implementation of the IV } \\
\boldsymbol{L}_{\infty}=50.1389 ; \boldsymbol{K}=0.7850 ; \boldsymbol{t}_{0}=0.1158 \\
S_{\text {otn_model }}=\frac{S_{\text {emodel }}}{S_{L}}=0.0269=2.69 \%\end{array}$ & $\begin{array}{l}\text { 4.1. Gulland-Holt method for estimation of } K \text { and } L_{\infty} \\
\boldsymbol{L}_{\infty}=50.1409 ; \boldsymbol{K}=0.7850 \\
\text { 4.2. Von Bertalanffy method for estimation of } t_{0} \\
\left(L_{\infty}=50.1409\right) \\
\boldsymbol{t}_{0}=0.0696\end{array}$ \\
\hline \multicolumn{2}{|l|}{ 5. Simulation data: $\boldsymbol{t}_{\text {sim }}$ and $\boldsymbol{L}_{15}=\boldsymbol{L}_{\text {sim }}+\boldsymbol{e}_{15}$} \\
\hline $\begin{array}{l}5.1 \text { After the executi } \boldsymbol{L}_{\infty}=50.8706 \text { on of the LSM } \\
\boldsymbol{L}_{\infty}=50.8706 ; \boldsymbol{K}=0.7590 ; \boldsymbol{t}_{0}=0.1285 \\
5.2 \text { After the } \boldsymbol{t}_{0}=0.0366 \text { implementation of the IV } \\
\boldsymbol{L}_{\infty}=50.8651 ; \boldsymbol{K}=0.7592 ; \\
S_{\text {otn_model }}=\frac{S_{\text {emodel }}}{S_{L}}=0.0460=4.60 \% \\
\boldsymbol{t}_{0}=0.1283\end{array}$ & $\begin{array}{l}\text { 5.1. Gulland-Holt method for estimation of } K \text { and } L_{\infty} \\
; \boldsymbol{K}=0.7590 \\
\text { 5.2. Von Bertalanffy method for estimation of } t_{0} \\
\left(L_{\infty}=50.8706\right)\end{array}$ \\
\hline \multicolumn{2}{|l|}{ 6. Simulation data: $\boldsymbol{t}_{\text {sim }}$ and $\boldsymbol{L}_{2}=\boldsymbol{L}_{\text {sim }}+\boldsymbol{e}_{2}$} \\
\hline $\begin{array}{l}6.1 \text { After the execution of the LSM } \\
\boldsymbol{L}_{\infty}=50.2648 ; \boldsymbol{K}=0.7521 ; \boldsymbol{t}_{0}=0.0680 \\
6.2 \text { After the implementation of the IV } \\
\boldsymbol{L}_{\infty}=50.2537 ; \boldsymbol{K}=0.7525 ; \boldsymbol{t}_{0}=0.0680 \\
S_{\text {otn_model }_{1}}=\frac{S_{\text {emodel }}}{S_{L}}=0.0193=1.93 \%\end{array}$ & $\begin{array}{l}\text { 6.1. Gulland-Holt method for estimation of } K \text { and } L_{\infty} \\
\boldsymbol{L}_{\infty}=50.2648 ; \boldsymbol{K}=0.7521 \\
\text { 6.2. Von Bertalanffy method for estimation of } t_{0} \\
\left(L_{\infty}=50.2648\right) \\
\boldsymbol{t}_{0}=0.0595\end{array}$ \\
\hline \multicolumn{2}{|l|}{ 7. Simulation data: $\boldsymbol{t}_{\text {sim }}$ and $\boldsymbol{L}_{3}=\boldsymbol{L}_{\text {sim }}+\boldsymbol{e}_{3}$} \\
\hline $\begin{array}{l}\text { 7.1 After the execution of the LSM } \\
\boldsymbol{L}_{\infty}=49.6445 ; \boldsymbol{K}=0.8100 ; \boldsymbol{t}_{0}=0.1165 \\
\text { 7.2 After the implementation of the IV } \\
\boldsymbol{L}_{\infty}=49.6637 ; \boldsymbol{K}=0.8092 ; \boldsymbol{t}_{0}=0.1164 \\
S_{\text {otn_model }_{-}}=\frac{S_{\text {emodel }}}{S_{L}}=0.0262=2.62 \%\end{array}$ & $\begin{array}{l}\text { 7.1. Gulland-Holt method for estimation of } K \text { and } L_{\infty} \\
\boldsymbol{L}_{\infty}=49.6445 ; \boldsymbol{K}=0.8100 \\
\text { 7.2. Von Bertalanffy method for estimation of } t_{0} \\
\left(L_{\infty}=49.6445\right) \\
\boldsymbol{t}_{0}=0.1043\end{array}$ \\
\hline
\end{tabular}


8.1 After the execution of the LSM

$\boldsymbol{L}_{\infty}=51.6138 ; \boldsymbol{K}=0.6796 ; \boldsymbol{t}_{0}=0.0233$

8.2 After the implementation of the IV

$\boldsymbol{L}_{\infty}=51.5260 ; \boldsymbol{K}=0.6822 ; \boldsymbol{t}_{0}=0.0328$

$S_{\text {otn_model }}=\frac{S_{\text {emodel }}}{S_{L}}=0.0583=5.83 \%$
8.1 Gulland-Holt method for estimation of $K$ and $L_{\infty}$ $\boldsymbol{L}_{\infty}=48.9194_{;} \boldsymbol{K}=0.8718$

8.2. Von Bertalanffy method for estimation of $t_{0}$

$\left(L_{\infty}=48.9194\right)$

$\boldsymbol{t}_{0}=0.2288$

9. Simulation data: ${ }_{\text {sim }}$ and $\boldsymbol{L}_{6}=\boldsymbol{L}_{\text {sim }}+\boldsymbol{e}_{6}$ graphical representation is depicted in Figure 11

9.1 After the execution of the LSM

$\boldsymbol{L}_{\infty}=54.8288 ; \boldsymbol{K}=0.6729 ; \boldsymbol{t}_{0}=0.2478$

9.2 After the implementation of the IV

$\boldsymbol{L}_{\infty}=54.7668 ; \boldsymbol{K}=0.6745 ; \boldsymbol{t}_{0}=0.2478$

$S_{\text {otn_ } \_ \text {model }}=\frac{S_{\text {emodel }}}{S_{L}}=0.2068=0.20 .68 \%$

9.1. Gulland-Holt method for estimation of $K$ and $L_{\infty}$

$\boldsymbol{L}_{\infty}=54.8288 ; \boldsymbol{K}=0.6729$

9.2. Von Bertalanffy method for estimation of $t_{0}$

$\left(L_{\infty}=54.8288\right)$

$\boldsymbol{t}_{0}=-0.1327$

10. Simulation Data: $\boldsymbol{t}_{01}=\boldsymbol{t}_{\text {sim }}+\boldsymbol{e}_{01}$ and $\boldsymbol{L}_{\text {sim }}$

10.1 After the execution of the LSM

$\boldsymbol{L}_{\infty}=50.0168_{;} \boldsymbol{K}=0.9079 ; \boldsymbol{t}_{0}=0.3052$

10.2 After the implementation of the IV

$\boldsymbol{L}_{\infty}=50.1028 ; \boldsymbol{K}=0.9043 ; \boldsymbol{t}_{0}=0.2995$

$S_{\text {otn_model }}=\frac{S_{\text {emodel }}}{S_{L}}=0.2353=23.87 \%$

10.1. Gulland-Holt method for estimation of $K$ and $L_{\infty}$

$\boldsymbol{L}_{\infty}=51.6138 ; \boldsymbol{K}=0.6796$

10.2. Von Bertalanffy method for estimation of $t_{0}$

$\left(L_{\infty}=51.6138\right)$

$\boldsymbol{t}_{0}=0.0118$

11. Simulation Data $\boldsymbol{t}_{05}=\boldsymbol{t}_{\text {sim }}+\boldsymbol{e}_{05}$ and $\boldsymbol{L}_{\text {sim }}$

11.1 After the execution of the LSM

$\boldsymbol{L}_{\infty}=11.0630 ; \boldsymbol{K}=-0.4741 ; \boldsymbol{t}_{0}=1.2886$

3.2 After the implementation of the IV

$\boldsymbol{L}_{\infty}=103.4981 ; \boldsymbol{K}=0.1124 ; \boldsymbol{t}_{0}=1.2886$

$S_{\text {otn_model }_{-}}=\frac{S_{\text {emodel }}}{S_{L}}=0.3192=31.92 \%$
11.1. Gulland-Holt method for estimation of $K$ and $L_{\infty}$

The model is proved statistically invalid

10.2. Von Bertalanffy method for estimation of $t_{0}$ 
Figure 9. Growth rate $\frac{\mathrm{dL}(\mathrm{t})}{\mathrm{dt}}$ functional relationship of $\mathrm{L}_{\mathrm{si}}(\mathrm{t})$; the $2^{\text {nd }}$ graph (at the bottom) represents $\mathrm{L}_{\operatorname{sim}}$ - the simulation data model and $\mathrm{L}_{\text {model }}$ - the model, obtained by implementing the parameters estimated by the Two-step LS and IV methodbased procedure in the absence of noise (added measurement error)
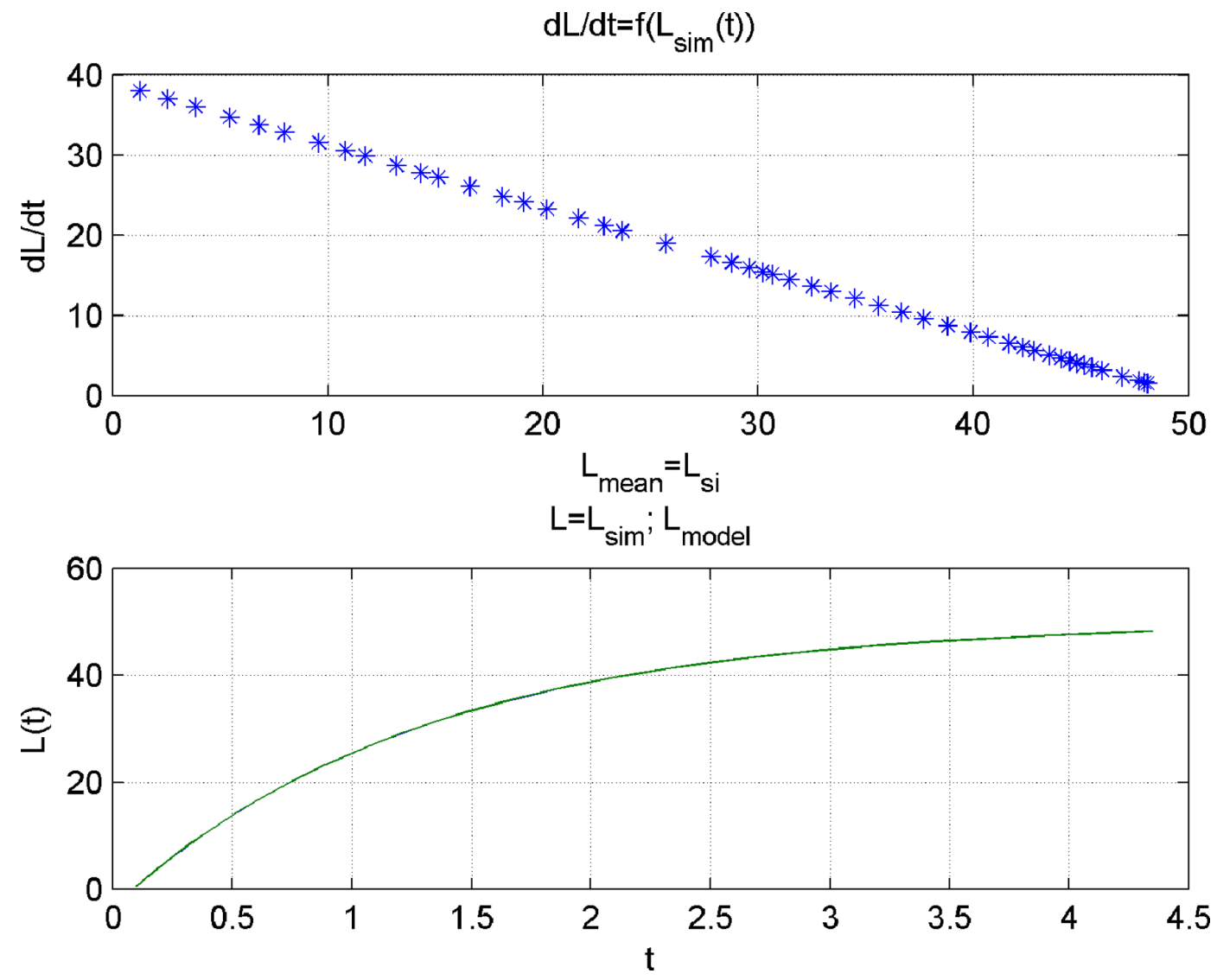
Figure 10. Growth rate $\frac{\mathrm{dL}(\mathrm{t})}{\mathrm{dt}}$ functional relationship of $\mathrm{L}_{\mathrm{si}}(\mathrm{t})$; the $2^{\text {nd }}$ graph (at the bottom) represents $\mathrm{L}_{\text {sim }}$-the simulation data model and $\mathrm{L}_{\text {model }}$ - the model, obtained by implementing the parameters estimated by the Two-step LS and IV methodbased procedure in the presence of noise (added measurement error) $\mathrm{e}_{2}$
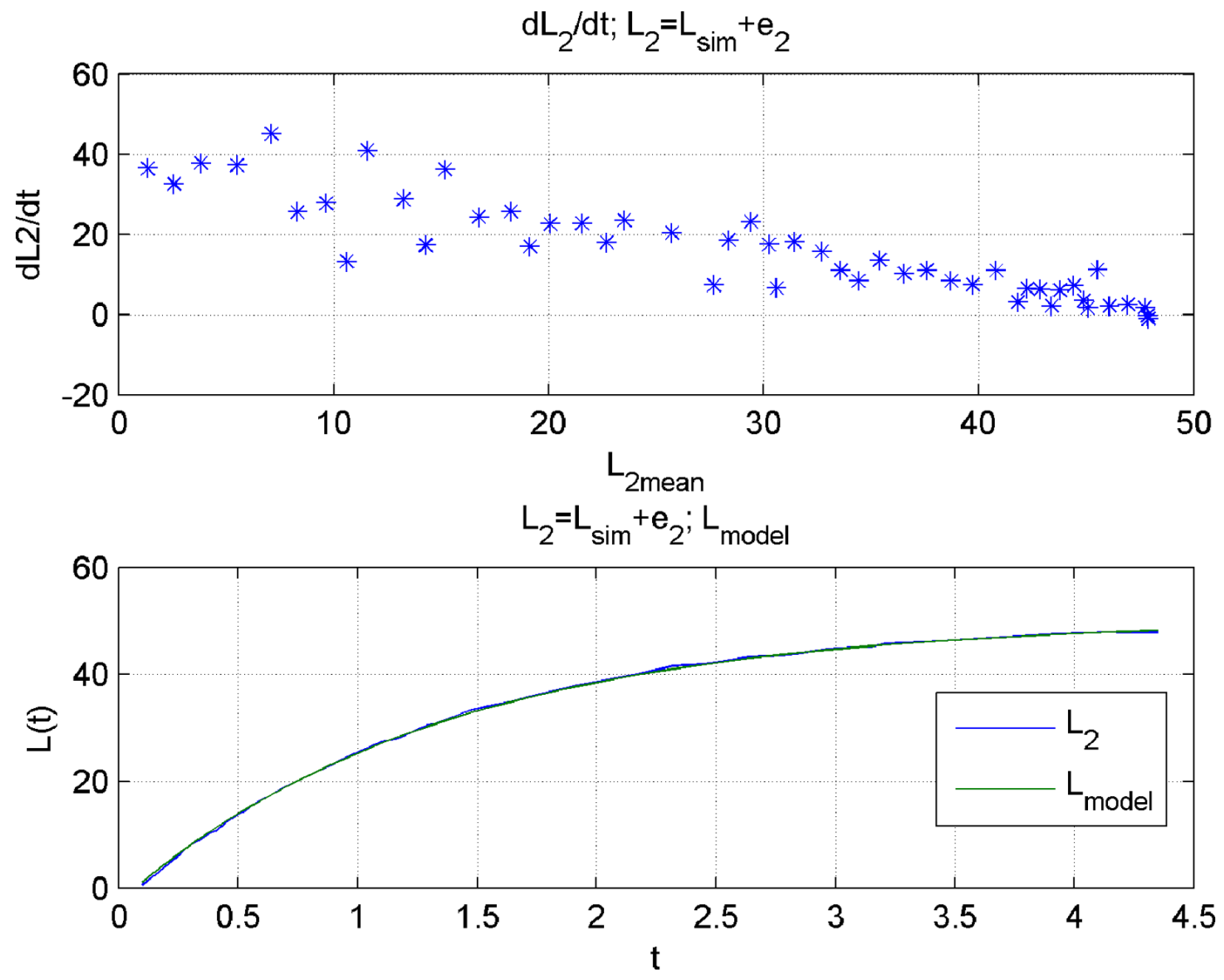
Figure 11. Growth rate $\frac{\mathrm{dL}(\mathrm{t})}{\mathrm{dt}}$ functional relationship of $\mathrm{L}_{\mathrm{si}}(\mathrm{t})$; the $2^{\text {nd }}$ graph (at the bottom) represents $\mathrm{L}_{\text {sim }}$ - the simulation data model and $\mathrm{L}_{\text {model }}$ - the model, obtained by implementing the parameters estimated by the Two-step LS and IV methodbased procedure in the presence of noise (added measurement error) $\mathrm{e}_{6}$
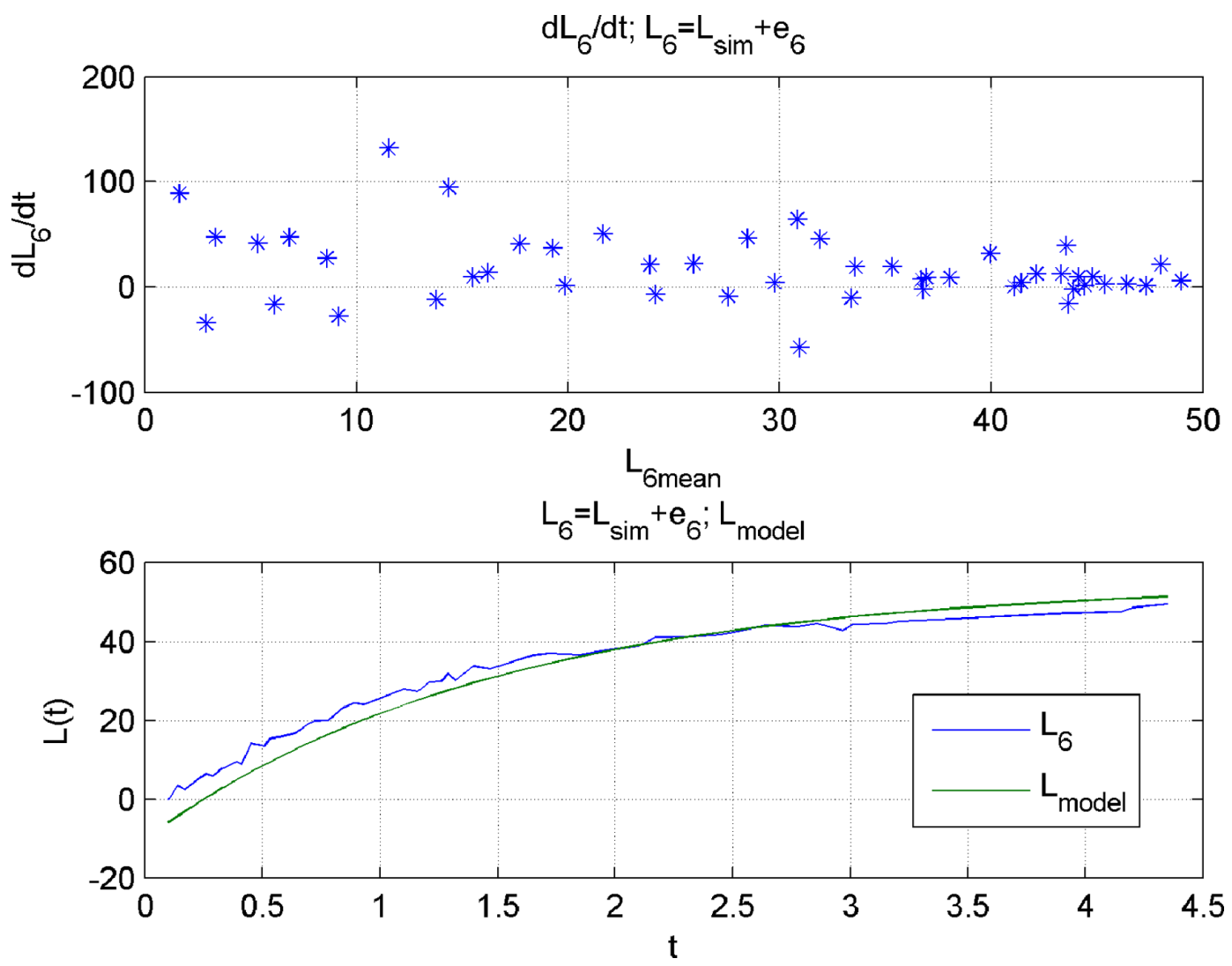


\section{REFERENCES}

Angrist, J., \& Krueger, A. (2001). Instrumental Variables and the Search for Identification: From Supply and Demand to Natural Experiments. The Journal of Economic Perspectives, 15(4), 69-85. doi:10.1257/jep.15.4.69

Bound, J., Jaeger, D. A., \& Baker, R. M. (1995). Problems with Instrumental Variables Estimation when the Correlation between the Instruments and the Endogenous Explanatory Variable is Weak. Journal of the American Statistical Association, 90(430).

Bowden, R. J., \& Turkington, D. A. (1984). Instrumental Variables. Cambridge, England: Cambridge University Press.

Bradistilov, G. (1961). Mathematics, Part 2, Mathematical analysis. Sofia: Tehnica.

Bretscher, O. (1995). Linear Algebra With Applications (3rd ed.). Upper Saddle River, NJ: Prentice Hall.

Cadima, E. L. (2003). Fish Stock Assessment Manual [technical paper]. FAO Fisheries.

Eykhoff, P. (1974). System Identification - Parameter and System Estimation. New York: John Wiley \& Sons.

Fuller, W. A. (1987). Measurement Error Models. John Wiley \& Sons. doi:10.1002/9780470316665

Genov, D. (2004). System identification Manual Lab. Varna: Technical University Varna.

Hoffman, J. D., \& Frankel, S. (2001). Numerical methods for engineers and scientists. Boca Raton, FL: CRC Press.

Lennart, L. (1999). System Identification - Theory For the User (2nd ed.). Upper Saddle River, N.J.: PTR Prentice Hall.

Melnikova, E. B. (2009). Definition of coefficients of the Bertalanffy Growth equation when regular measurements are lacking, Biological resources of the White Sea and inland waters of the European North. In Proceedings of the XXVIII International Conference, October 5-8 (pp. 353-356).

Soderstrom, T., \& Stoica, P. (1989). System Identification. Upper Saddle River, N.J.: Prentice Hall.

Sparre, P., \& Venema, S. C. (1998). Introduction to Tropical Fish Stock Assessment - Part 1: Manual [technical paper]. FAO Fisheries.

Stigler, S. M. (1981). Gauss and the Invention of Least Squares. Annals of Statistics, 9(3), 465-474. doi:10.1214/ aos/1176345451

Stock, J. H., \& Trebbi, F. (2003). Retrospectives: Who Invented Instrumental Variable Regression? The Journal of Economic Perspectives, 17(3), 177-194. doi:10.1257/089533003769204416

Walter, E., \& Pronzato, L. (1997). Identification of Parametric Models from Experimental Data. Springer. 


\section{APPENDIX A: MATLAB-ENVIRONMENT SCRIPT}

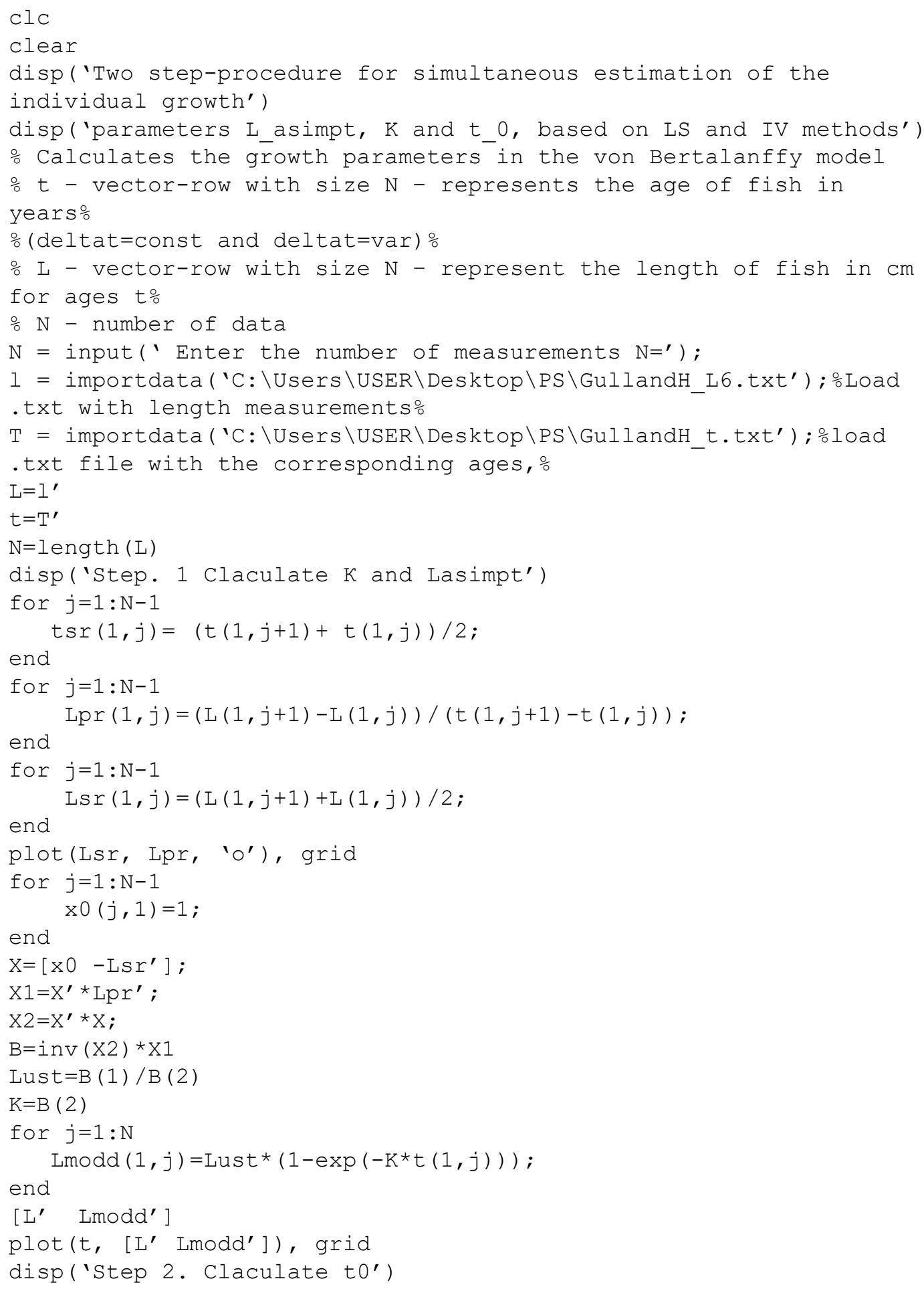




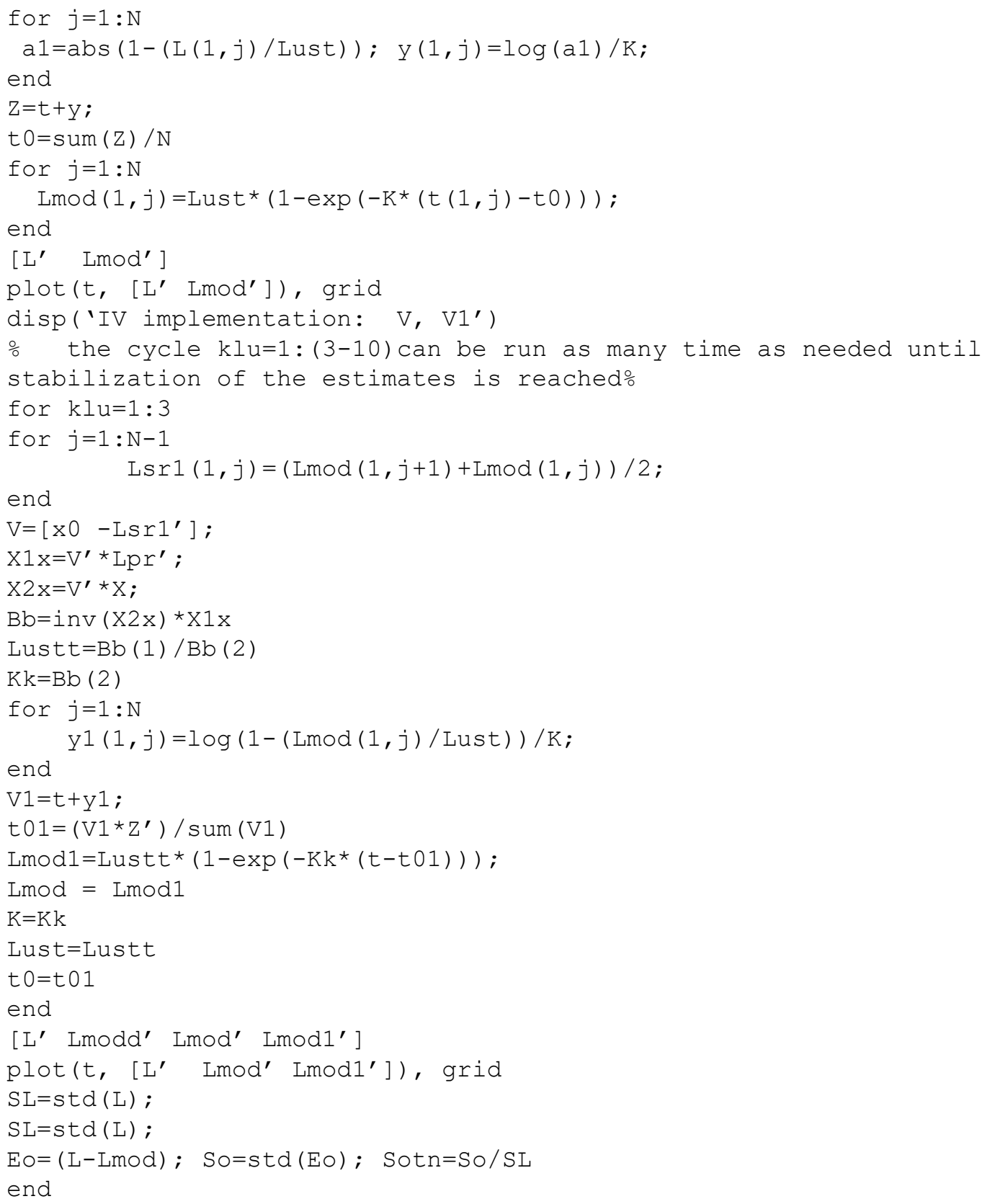

\section{APPENDIX B: CONTENT OF THE PROGRAM SCRIPT}

$\mathrm{clc}$

clear

disp('1. Gulland and Holt Method for estimation of Lasimpt and $\mathrm{K}$ ') 
$r=$ input (' Enter the number of measurements $r={ }^{\prime}$ );

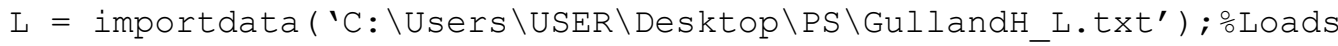

.txt file with Length measurements to form the input vector for

the regression analysiso

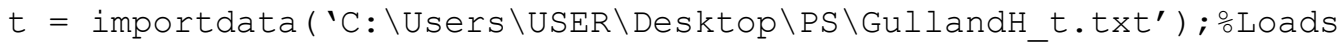

.txt file with the corresponding age data\%

$\mathrm{n}=\mathrm{r}-1$

for $i=1: n$

deltat $(i)=t(i+1)-t(i) ; \circ c a l c u l a t e s$ deltato

deltaL $(i)=\mathrm{L}(i+1)-\mathrm{L}(i)$; $\frac{\circ}{c}$ alculates the increment in length for step deltato

$$
y(i)=\operatorname{deltaL}(i) / \operatorname{deltat}(i) \text {; }
$$

end

$\mathrm{Y}=\mathrm{Y}^{\prime}$

oCalculates the mean length for interval deltat and forms the independent variable $x$ for the regression analysiso

for $i=1: n$

Lmean $(i)=((\mathrm{L}(\mathrm{i})+\operatorname{deltaL}(\mathrm{i}))+\mathrm{L}(\mathrm{i})) / 2$;

end

$\mathrm{x}=$ Lmean'

disp ( $* * *$ a.Regression analysis $* * * \prime)$

$\mathrm{x} 0=\operatorname{ones}(\mathrm{n}, 1)$;

$\mathrm{F}=[\mathrm{x} 0, \mathrm{x}] ;$; Forms the regression matrix응

$\mathrm{FF}=\left(\mathrm{F}^{\prime} \star \mathrm{F}\right)$;

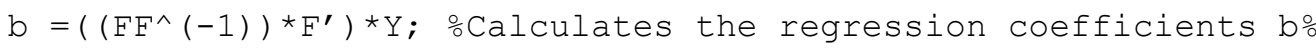

beta $=\mathrm{b}^{\prime}$;

$\mathrm{b} 0=\operatorname{beta}(1,1)$;

$\mathrm{b} 1=\operatorname{beta}(1,2)$;

disp('Check the condition number of matrix FF:')

Cond_FF $=$ cond $(F F) \div$ determines the condition factor of the information matrix FFl, which forms the covariance matrix, once inverted - if the condition factor cond(FF) >10-10^2 it is recommended to run the analysis with normalized/standardized variables\%

maxval $=10$;

if (Cond_FF $>$ maxval)

disp ('Cond $\mathrm{FF}>10==>$ ')

disp('Run the regression analysis with standardized/Normalized variables')

xmean $=$ mean $(x) ; \circ$ Calculates the mean value of $x=$ Lmean $\%$

ymean $=$ mean $(Y)$; $\circ$ Calculates the mean value of $y=$ deltaL/deltat $\%$

$\mathrm{sX}=\operatorname{std}(\mathrm{x}) ; \circ \mathrm{Calculates}$ the standard deviation of $\mathrm{x} \%$

Xnorm $=(\mathrm{x}$-xmean $) / \mathrm{sX}$; ㅇtandardizes $\mathrm{x}$ (the observed/measured values) $\%$

$\mathrm{fo}=\operatorname{ones}(\mathrm{n}, 1)$;

Fnorm $=[f 0$, Xnorm $]$; 으orms the regression matrix

FFnorm $=$ Fnorm' ${ }^{*}$ Fnorm;

disp('Condition factor of FFnorm')

Cond_norm $=$ cond(FFnorm) $\frac{\circ}{\circ}$ Claculates the condition factor of the matrix FFnormo 
oformed by using the standardized $x$ values\%

$\mathrm{B}=\left(((\right.$ FFnorm $) \wedge(-1)){ }^{\wedge}$ Fnorm' $) * \mathrm{Y}$;

$\mathrm{Bl}=\mathrm{B}^{\prime} ;$

disp('Regression coefficient bon - standardized variables')

$\mathrm{b} 0 \mathrm{n}=\operatorname{Bl}(1,1)$

disp('Regression coefficient bln - standardized variables')

$\mathrm{b} 1 \mathrm{n}=\mathrm{Bl}(1,2)$

disp ('Regression coefficient B0 - model')

$\mathrm{BO}=(\mathrm{b} 0 \mathrm{n}-((\mathrm{b} 1 \mathrm{n} * \mathrm{xmean}) / \mathrm{sX}))$

disp('Regression coefficient B1 - model')

$\mathrm{B} 1=\mathrm{b} 1 \mathrm{n} / \mathrm{sX}$

disp('Calculates the estimation of the dependent variable $y_{-}$ hat $=$ GrR by using the values calculated for the regression

coefficients yhat $=:^{\prime}$ )

yhat $=\mathrm{B} 0+\mathrm{B} 1{ }^{*} \mathrm{X}$

e $=$ Y-yhat

disp('Calculates the model error e:')

scatter $(\mathrm{X}, \mathrm{Y})$; grid

title('Regression analysis(Plot (y_m_e_a_s, $\mathrm{x}$ ) и (y_h_a_t, $\mathrm{x}$ )')

hold on

$\mathrm{y} 1$ = yhat;

plot $(x, y 1)$

hold off

else

disp ('Condition number of matrix $\mathrm{FF}<10$ ')

$\mathrm{b} 0=\operatorname{beta}(1,1)$

disp('Regression coefficient b0=:')

$\mathrm{b} 1=\operatorname{beta}(1,2)$

disp('Regression coefficient b1=:')

disp('Calculates the estimation of the dependent variable $y_{-}$ hat $=$ GrR by using the values calculated for the regression

coefficients yhat $=:^{\prime}$ )

yhat $=\mathrm{b} 0+\mathrm{b} 1 * \mathrm{x}$

disp('Calculates the model error e:')

e $=$ Y-yhat

scatter $(\mathrm{X}, \mathrm{Y})$; grid

title ('Regression analysis (Plot (y_m_e_a_s, $x$ ) и (y_h_a_t, $x$ )') hold on

y1 = yhat;

plot $(\mathrm{x}, \mathrm{y} 1)$

hold off

end

disp ('***b. Model Statistical analysis***')

QO $=\operatorname{sum}\left((Y-y h a t) \cdot{ }^{\wedge} 2\right) ;$ oCalculates the partition of the sum of squares\%

$\mathrm{Q}=\operatorname{sum}\left((\mathrm{Y}-\mathrm{ymean}) \cdot{ }^{\wedge} 2\right) ; \circ \mathrm{Calculates}$ the total sum of squares $\%$

Qcalc $=$ Qo/Q;

disp('Calculate the correlation coefficient $R^{\prime}$ )

$R=\operatorname{sqrt}((1-Q \operatorname{cal} C))$

disp('F-test the $R$ value and model workability $F='$ ) 
$\mathrm{F}=\left(\mathrm{R}^{\wedge} 2 *(\mathrm{n}-2)\right) /\left(\left(1-\mathrm{R}^{\wedge} 2\right) * 1\right) \div \mathrm{FC}$ is found in F-test distribution

table to be compared\%

owith the calculated value F>FCr\%

Fcr = input ('Enter table value for Fcr=:');

if $(\mathrm{F}>\mathrm{FCr})$

disp('The model is proved functional and can be used to

predict the behavior of the BO under analysis')

figure

scatter $(\mathrm{X}, \mathrm{Y})$; grid

$\left.\left(y+h \_a+t, x\right)^{\prime}\right)$

title('Regression analysis(Plot (y_m_e_a_s, $x$ ) и

$\mathrm{y} 1=$ yhat;

hold on

plot $(\mathrm{x}, \mathrm{y} 1)$

hold off

if (Cond_FF $>$ maxval)

disp('Claculate the growth parameters $\mathrm{K}$ и L_asimpt

by using the calculated regression coefficients')

$$
\begin{aligned}
& \text { disp }(' K=: ') \\
& K=-B 1 \\
& a=B 0 ; \\
& \text { disp ('L_asimpt }=: ' \text { ') } \\
& \text { Lasimpt }=-(\mathrm{a} / \mathrm{B} 1
\end{aligned}
$$

else

disp('The model is proved non-functional and respectively cannot be used to predict the BO behavior')

end

disp('2. Von Bertalanffy method for estimation of $\mathrm{K}$ и to')

disp ('***Provides estimates of $K$ and to if Lasimpt is known***') $r=$ input (' Enter the number of measurements (observations) $r={ }^{\prime}$ );

Lasimpt = input (' Enter value for Lasimpt=');

$x=t$

$y=-\log (1-($ L/Lasimpt $))$ Forms the output vector $y$ for the regression analysis\%

disp ( $* * * a$.Regression analysis***')

$\mathrm{x} 0=\operatorname{ones}(r, 1)$;

$\mathrm{F}=[\mathrm{x} 0, \mathrm{x}] ;$; Forms the regression matrix응

$\mathrm{FF}=\left(\mathrm{F}^{\prime} \star \mathrm{F}\right) ;$

$\mathrm{b}=\left(\left(\mathrm{FF}^{\wedge}(-1)\right){ }^{*} \mathrm{~F}^{\prime}\right){ }^{*} \mathrm{Y}$; $\frac{\circ}{\mathrm{C}} \mathrm{Calculates}$ the regression koefficients $\mathrm{b}$ _i\%

beta $=\mathrm{b}^{\prime}$;

$\mathrm{b} 0=\operatorname{beta}(1,1)$;

$\mathrm{b} 1=\operatorname{beta}(1,2)$;

disp ('Check the condition number of the matrix FF:')

Cond_FF = cond $(\mathrm{FF}))$ \% determines the condition factor of the information matrix FFl, which forms the covariance matrix, once inverted - if the condition factor cond(FF) >10-10^2 it is recommended to run the analysis with normalized/standardized variables\%

maxval $=10$; 
if (Cond_FF> maxval)

disp ('Cond $\mathrm{FF}>10==>$ ')

disp('Run the regression analysis with standardized/Normalized variables')

xmean $=$ mean $(\mathrm{x})$; $\%$ Calculates the mean value of $\mathrm{x}=\mathrm{t} \%$

ymean $=$ mean $(y) ;$ ocalculates the mean value of $y=-\log (1-(\mathrm{L} /$ Lasimpt)) $\%$

$\mathrm{sX}=\operatorname{std}(\mathrm{x}) ; \%$ Calculates the standard deviation of $\mathrm{x} \%$

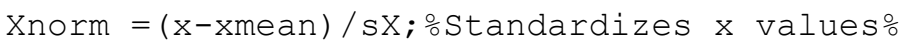

$\mathrm{fo}=\operatorname{ones}(r, 1)$;

Fnorm $=[$ f0, Xnorm $]$; ․Forms the regression matrix by using the standardized $\mathrm{x}$ values응

FFnorm = Fnorm'* Fnorm;

disp('Condition number of matrix FFnorm')

Cond norm $=$ cond $($ FFnorm

$\mathrm{B}=\left(\overline{(}(\text { FFnorm })^{\wedge}(-1)\right){ }^{*}$ Fnorm $\left.{ }^{\prime}\right){ }^{*}$;

$\mathrm{Bl}=\mathrm{B}^{\prime} ;$

disp('Regression coefficient bon - standardized variables')

$\mathrm{b} 0 \mathrm{n}=\mathrm{Bl}(1,1)$

disp('Regression coefficient bln - standardized variables')

$\mathrm{b} 1 \mathrm{n}=\mathrm{Bl}(1,2)$

disp('Regression coefficient BO - model')

$\mathrm{BO}=(\mathrm{b} 0 \mathrm{n}-((\mathrm{b} 1 \mathrm{n} * \mathrm{xmean}) / \mathrm{sX}))$

disp('Regression coefficient B1 - model')

$\mathrm{B} 1=\mathrm{b} 1 \mathrm{n} / \mathrm{sX}$

disp('Calculates the estimation of the dependent variable $y$ hat by using the values calculated for the regression coefficients yhat $=$ : ')

yhat $=\mathrm{B} 0+\mathrm{B} 1{ }^{*} \mathrm{X}$

disp('Calculates the model error e e:')

e $=y$-yhat

else

disp ('The condition number of matrix $\mathrm{FF}<10$ ')

disp ('Regression coefficient b0=:')

$\mathrm{b} 0=\operatorname{beta}(1,1)$

disp('Regression coefficient $\mathrm{b} 1=:^{\prime}$ )

$\mathrm{b} 1=\operatorname{beta}(1,2)$

disp('Calculates the estimation of the dependent

variable y hat by using the values calculated for the regression

coefficients yhat $=$ : yhat $=:^{\prime}$ )

yhat $=\mathrm{b} 0+\mathrm{b} 1 * \mathrm{t}$

disp ('Claculates the model error e:')

$e=y$-yhat

end

disp $(* * *$ b. Model statistical analysis***')

Q० $=\operatorname{sum}\left((y-y h a t) \cdot{ }^{\wedge} 2\right) ; \%$ Calculates the partition of

the sum of squares \%

ymean=mean $(y)$;

$\mathrm{Q}=\operatorname{sum}\left((\mathrm{y}-\mathrm{ymean}) \cdot{ }^{\wedge} 2\right) ; \circ$ Calculates the total sum of 


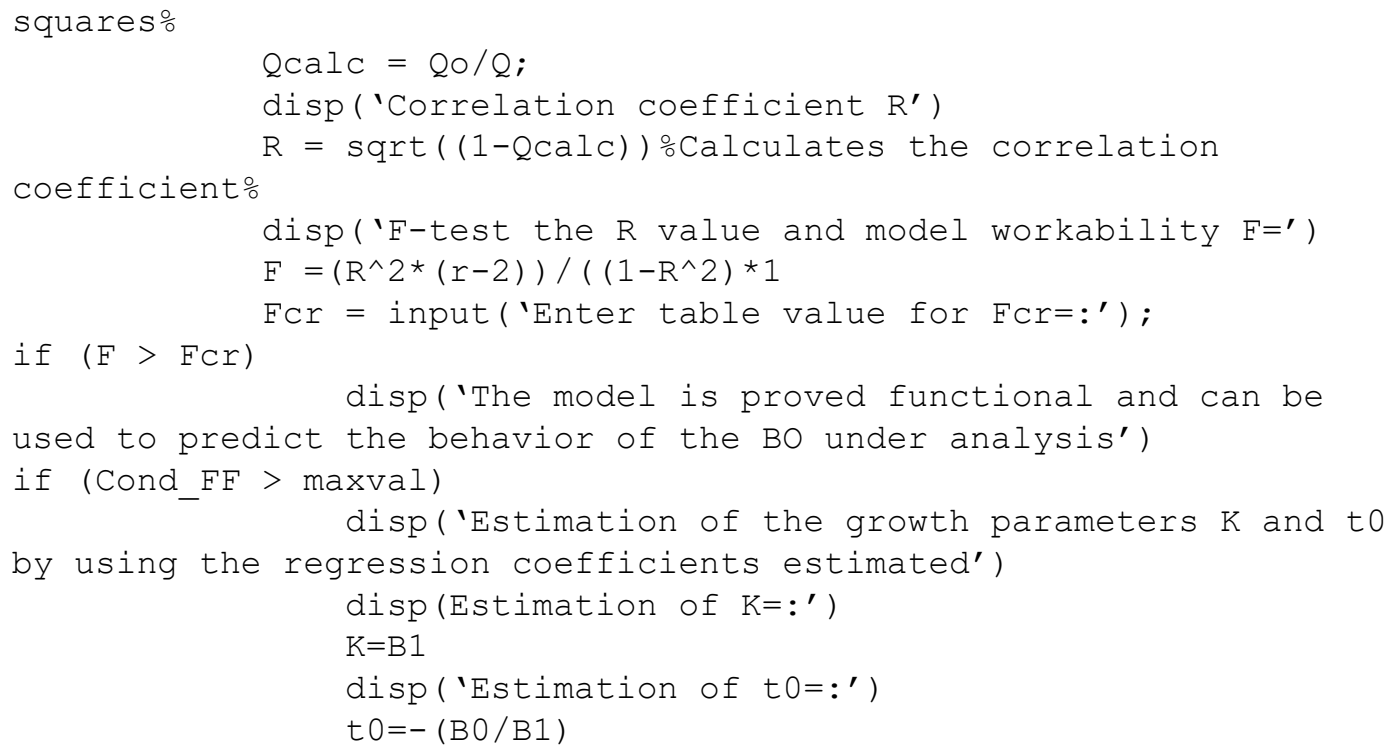

Ivelina Yordanova Zlateva is educated in Automation in 2000 and later on in 2009 in Industrial management. In 2016 assigned for PhD course at the Technical University of Varna. Her PhD work is interdisciplinary related to: "Engineering methods and their application for assessment and sustainable management of living marine resources in the Black sea." Main research area: engineering methods application in marine living resources management Application of Engineering methods in Biostatistics Matlab-programming. Recent publications: MATLAB-Based Standalone Application for Estimation of Growth and Mortality Parameters of Fish; MATLAB-Based Stochastic Modeling. Distribution Analysis of Commercial Fishery Length-Frequency Samples Taken From the Black Sea (Bulgaria); Matlab-Based Length/Weight Relationship Analysis of Commercial Fishery Samples taken from the Black Sea (Bulgaria); Environmental Management Ecosystem vs. Engineering System Theory Approach Modeling and Analysis: Risk Management System as a Managerial Tool.

Nikola Nikolov, Education: 2009 PhD, Topic: "Research of algorithms for the synthesis of modular adaptive condition regulators", 1989 Engineer Master, TU-Varna, specialization "Automation of Production", topic of the diploma thesis "Development of a system for controlling the electric drive of underwater equipment". Academic positions: January 2016 - Head of the Department of Automation of Production at TU-Varna. February 2013 - Associate Professor, Department of Automation of Production at TU-Varna. Main Research areas: Process control; Technological Processes Control with State Controllers; Adaptive Observation; Engineering Practices in Marine Resource Management. Selected Publications: Progressive Engineering Practices in Marine Resource Management; Enviromental Management Ecosystems vs. Engineering System Theory Approach Modeling and Analysis: Risk Management System as a Managerial Tool; Adaptive State Observer Development Using Recursive Extended Least-Squares Method; Development of an Algorithm for Modal Control ofSISO Linear Time-Invariant Discrete Systems; Discrete Adaptive Real-Time State Observer Development Using Least-Squares Method. 